\title{
NONLINEAR PRECONDITIONING STRATEGIES FOR TWO-PHASE FLOWS IN POROUS MEDIA DISCRETIZED BY A FULLY IMPLICIT DISCONTINUOUS GALERKIN METHOD*
}

\author{
LI LUO ${ }^{\dagger}$, XIAO-CHUAN CAI ${ }^{\ddagger}$, AND DAVID E. KEYES ${ }^{\dagger}$
}

Abstract. We consider numerical simulation of two-phase flows in porous media using implicit methods. Because of the complex features involving heterogeneous permeability and nonlinear capillary effects the nonlinear algebraic systems arising from the discretization are very difficult to solve. The traditional Newton method suffers from slow convergence in the form of a long stagnation or sometimes does not converge at all. In the paper, we develop some nonlinear preconditioning strategies for the system of two-phase flows discretized by a fully implicit discontinuous Galerkin method. The preconditioners identify and approximately eliminate the local high nonlinearities that cause the Newton method to take small updates. Specifically, we propose two elimination strategies, one is based on exploring the unbalanced nonlinearities of the pressure and the saturation fields, and the other is based on identifying certain elements of the finite element space that have much higher nonlinearities than the rest of the elements. We compare the performance and robustness of the proposed algorithms with an existing single-field elimination approach and the classical inexact Newton method with respect to some physical and numerical parameters. Experiments on three-dimensional porous media applications show that the proposed algorithms are superior to the other methods in terms of the robustness and parallel efficiency.

Key words. Two-phase flow in porous media, inexact Newton, nonlinear preconditioning, fully implicit, discontinuous Galerkin, parallel computing

AMS subject classifications. 76S05, 49M15, 76D05

1. Introduction. Simulation of two-phase flows in porous media plays a vital role in hydrology and petroleum reservoir engineering. The mathematical model addressing the problem is a coupled system of time-dependent nonlinear PDEs, including the Darcy's law, the equation of mass conservation for each phase, the constraint of the saturations, and the dependency of capillary pressure on the wetting saturation. The complexity of this model lies in the interaction of various modeling features, particularly, the heterogeneous permeability of high contrast, strong nonlinearity of relative permeability functions, and spatially varying capillary pressure [49]. Extra difficulties are induced by complex geometry, faults, channels and voids. For general reservoir models with such complexities, it is important to design accurate, fast and robust solution algorithms to obtain reliable simulation results.

A large number of numerical methods have been developed to model two-phase flows in heterogeneous media. One popular solution algorithm used in practice is IMPES (implicit pressure and explicit saturation) [9, 10] in which the pressure equation is first solved and then the saturation is updated by an explicit time-stepping scheme. Since the saturation often changes faster than the pressure, in general, several small time steps are performed for the saturation equation immediately after a large time step for the pressure equation. Enhanced versions of IMPES have been studied in $[1,11,30,45]$ to improve the accuracy and stability by using a semi-implicit scheme for the saturation equation or by introducing a number of iterations in a single

\footnotetext{
* Submitted to the editors DATE.

Funding: The first author is supported in part by NSFC grant 11701547 and NSFC-RGC joint research grant N-HKUST620/15.

†Extreme Computing Research Center, King Abdullah University of Science and Technology, Thuwal 23955-6900, Saudi Arabia (li.luo@kaust.edu.sa, david.keyes@kaust.edu.sa).

${ }^{\ddagger}$ Department of Mathematics, University of Macau, Macau, China (xccai@um.edu.mo).
} 
pressure-saturation time step. It is believed that the most stable scheme for multiphase flows is the fully implicit method in which all the coupled nonlinear equations are solved simultaneously in a single time step [3, 13, 16, 41]. Theoretically, the fully implicit method yields unconditional stability so that it often allows using a much larger time step size than the IMPES-type methods [31, 41, 49, 50, 52]. In our work, we introduce a fully implicit discontinuous Galerkin finite element scheme for the discretization of the two-phase flow problem. The discontinuous Galerkin (DG) method $[42,45]$ is attractive because of its flexibility in describing unstructured domains by using higher-order approximation functions, which generally provides higher resolution in the vicinity of sharp fronts of the saturation compared to lower-order methods, such as the cell-centered finite volume method [40]. Other advantages of DG include the robustness of the method for equations with discontinuous coefficients and the local mass conservation property. We refer to $[3,16,38]$ for more details of the fully implicit DG discretization for modeling two-phase flows in porous media.

The price to pay for using a fully implicit method is that a large sparse nonlinear system of equations has to be solved at each time step. Hence, it is challenging and crucial to design robust and efficient nonlinear solvers for the resultant algebraic systems. Efforts have been made to employ Newton's method and its variants to solve the two-phase flow problems $[13,41,49,50,51]$. The family of inexact Newton methods [15] is popular and has a rapid local convergence rate under certain conditions. A straightforward application of an inexact Newton method to the fully implicit simulation of two-phase flows works well for relatively simple problems [47], but for many practical problems it results in problematic convergence. The difficulty arises from the unbalanced nonlinearities generated by issues such as: discontinuity of permeability coefficients, vast variation of fluid properties, strong capillary effects with limited spatial extent, complex source terms, and corner/fault/void singularities. In such cases, some of the equations are more difficult to solve than the others in the system and an inexact Newton method may suffer from slow convergence in the form of a long stagnation, or not converge at all. To overcome this difficulty, a conventional treatment is to reduce the time step size substantially, but this detracts from the natural merit of fully implicit methods, especially for long time simulation at large scales.

Recently, a new class of algorithms, nonlinear preconditioning, has been shown useful in enhancing the robustness and efficiency of a Newton-like method. The idea of nonlinear preconditioning is to balance the nonlinearities of the overall system by removing local high nonlinearities that cause the Newton method to take small updates, so that fast convergence can be realized. Similar to linear preconditioning, nonlinear preconditioning is naturally classified as either 'left' or 'right'. Left nonlinear preconditioners introduce a set of small nonlinear problems that are less nonlinearly stiff than the original problem and still offer good convergence to the solution. The additive Schwarz preconditioned inexact Newton algorithm (ASPIN) [7, 23, 24, 25] belongs to this class, where solutions of nonlinear subdomain problems are solved and then combined together using an additive Schwarz framework. A multiplicative Schwarz version of ASPIN, named MSPIN, was introduced in [33, 35] for two-dimensional (2D) incompressible flows and two-phase flows in porous media [36].

Left nonlinear preconditioning changes the original nonlinear system to a more balanced one, and then solves the new system using a Newton-like method. In contrast, right nonlinear preconditioning such as nonlinear elimination (NE) [8] does not change the nonlinear function but modifies the unknown variables of the original system, which can be viewed as an inner correction step to provide a new starting point 
for the Newton iteration. Therefore, right preconditioning is more implementationfriendly compared to the left version. The NE algorithm is applied in the intermediate Newton solution to remove or reduce local high nonlinearities such that a high-quality Newton direction is obtained for the new iteration. How to identify the bad components in the nonlinear system is critical to the success of the NE preconditioner. Several strategies have been proposed recently. Hwang et al. [25, 26] used a physicsbased approach for transonic full potential problems. Huang et al. [22] and Yang et al. [51] applied a point-wise approach to eliminate the components associated with certain mesh points that produce the local high nonlinearities for 2D multi-component systems. Yang et al. [49, 50, 52] proposed a field-based component-wise approach to eliminate the components associated with some field variables, i.e., the saturation field in two-phase flows. More recently, Luo et al. [37, 39] extended the NE preconditioner to incompressible flow problems in three dimensions.

In practice, designing effective elimination strategies for the time-dependent twophase flow problem is not trivial, since the saturation interacts with the pressure, and the dynamics of saturation is significantly affected by the heterogeneous media. In this paper, considering a system of two-phase flows discretized by the fully implicit DG finite element scheme, we propose two new elimination strategies, respectively, a multiplicative field-split approach and a coupled element-block approach, to accelerate the Newton iteration. We compare numerically the robustness and efficiency of the proposed methods with the field-based component-wise method $[49,50]$ and the classical inexact Newton method for some nonlinearly difficult problems. In our implementation, we embed the nonlinear preconditioning step in an overlapping domain decomposition framework $[5,29,44]$ so that the overall method can be parallelized on machines with a large number of processor cores.

The paper is organized as follows. In section 2, the system of incompressible two-phase flow in porous media is presented and followed by a fully implicit DG finite element discretization. In section 3, the nonlinear elimination preconditioned inexact Newton method with different elimination strategies is presented in detail. Numerical experiments for two-phase flows in homogeneous and heterogeneous medias are provided in section 4 . The robustness and efficiency of the proposed methods are comprehensively studied and compared in this section. Some concluding remarks are given in section 5 .

\section{Mathematical model and discretization.}

2.1. Governing equations. Let $\Omega$ be a bounded porous medium in $\mathbb{R}^{3}$. The flow of the wetting phase (i.e., water) and non-wetting phase (i.e., oil) in $\Omega$ is governed by Darcy's law and the equation of mass conservation for each phase. We denote by the subscript $\alpha=w$ and $\alpha=n$ the wetting and non-wetting phase, respectively. The Darcy velocity for each phase is determined by

$$
\mathbf{u}_{\alpha}=-\lambda_{\alpha} \mathbf{K}\left(\nabla p_{\alpha}-\rho_{\alpha} g \nabla D\right), \quad \alpha=w, n,
$$

and the saturation equation for each phase satisfying the mass conservation is given by

$$
\phi \frac{\partial s_{\alpha}}{\partial t}+\nabla \cdot \mathbf{u}_{\alpha}=q_{\alpha}, \quad \alpha=w, n,
$$

where $\mathbf{u}_{\alpha}, s_{\alpha}, p_{\alpha}, \rho_{\alpha}, q_{\alpha}$ are, respectively, the velocity, saturation, pressure, density, and source of phase $\alpha . \phi$ is the porosity of the porous media and $\mathbf{K}$ is the absolute 
permeability tensor. For heterogenous porous media, $\phi$ and $\mathbf{K}$ can vary over several orders of magnitude and can be discontinuous in space. $g$ is the gravitational acceleration constant, and $D$ is the depth at position $(x, y, z)$. The mobility function $\lambda_{\alpha}$ is a ratio of the relative permeability $k_{r \alpha}\left(s_{w}\right)$ and the viscosity $\mu_{\alpha}$,

$$
\lambda_{\alpha}=\frac{k_{r \alpha}\left(s_{w}\right)}{\mu_{\alpha}}, \quad \alpha=w, n .
$$

The saturations of the two phases are constrained by

$$
s_{w}+s_{n}=1 .
$$

The relation between the wetting and non-wetting phase pressures is described by the capillary pressure $[10,21]$,

$$
p_{c}\left(s_{w}\right)=p_{n}-p_{w} .
$$

Substituting (2.1), (2.3), and (2.4) to (2.2), the two-phase conservative formulation reads

$$
\begin{aligned}
& -\phi \frac{\partial s_{w}}{\partial t}-\nabla \cdot\left(\lambda_{n} \mathbf{K}\left(\nabla p_{w}+\nabla p_{c}-\rho_{n} g \nabla D\right)\right)=q_{n}, \quad \text { in } \Omega, \\
& \phi \frac{\partial s_{w}}{\partial t}-\nabla \cdot\left(\lambda_{w} \mathbf{K}\left(\nabla p_{w}-\rho_{w} g \nabla D\right)\right)=q_{w}, \quad \text { in } \quad \Omega .
\end{aligned}
$$

Boundary conditions and initial condition are required to close the system. Let $\partial \Omega=$ $\Gamma_{\text {in }} \cup \Gamma_{\text {out }} \cup \Gamma_{0}$, where $\Gamma_{\text {in }}$ denotes the inlet boundary, $\Gamma_{\text {out }}$ denotes the outlet boundary, and $\Gamma_{\text {in }} \cap \Gamma_{\text {out }}=\emptyset . \Gamma_{0}=\partial \Omega \backslash\left\{\Gamma_{\text {in }} \cup \Gamma_{\text {out }}\right\}$ is the impermeable boundary. The boundary conditions are stated as:

$$
\begin{array}{ll}
\mathbf{u}_{w} \cdot \mathbf{n}=f_{w}^{\text {in }}, \mathbf{u}_{n} \cdot \mathbf{n}=f_{n}^{\text {in }}, & \text { on } \Gamma_{\text {in }}, \\
p_{w}=p_{w}^{\text {out }}, \lambda_{n} \mathbf{K} \nabla p_{c} \cdot \mathbf{n}=0, & \text { on } \Gamma_{\text {out }}, \\
\mathbf{u}_{w} \cdot \mathbf{n}=0, \mathbf{u}_{n} \cdot \mathbf{n}=0, & \text { on } \Gamma_{0},
\end{array}
$$

where $\mathbf{n}$ is the unit outward normal vector, $f_{w}^{i n}$ and $f_{n}^{i n}$ are given flow rates at the inlet. The initial condition is given by

$$
\left.s_{w}\right|_{t=0}=s_{w}^{0}, \quad \text { in } \Omega .
$$

The equations are coupled nonlinearly through the relative permeability and the capillary pressure, which are given by $([21])$ :

$$
k_{r w}\left(s_{w}\right)=s_{e}^{\beta}, \quad k_{r n}\left(s_{w}\right)=\left(1-s_{e}\right)^{\beta}, \quad p_{c}\left(s_{w}\right)=-\bar{B}_{c} \log \left(s_{e}\right),
$$

where $\beta, \bar{B}_{c}$ are positive parameters and $s_{e}$ is the effective saturation defined as $s_{e}=\left(s_{w}-s_{r w}\right) /\left(1-s_{r w}-s_{r n}\right)$. Here $s_{r w}$ and $s_{r n}$ are residual saturations for the wetting and non-wetting phases.

2.2. Fully implicit discontinuous Galerkin finite element discretization. In this paper, we solve the system of (2.5) and (2.6) simultaneously using a fully coupled approach. The discretization is based on a backward Euler scheme in time and an upwind non-symmetric interior penalty Galerkin (NIPG [16, 38]) finite element method in space. 
Let $\Omega_{h}=\{E\}$ be a quasi-uniform mesh of $\Omega$ consisting of $N_{E}$ elements. We denote by $\Gamma_{h}$ the set of faces in $\Omega_{h}$ and by $e$ the face shared by two elements $E_{a}$ and $E_{b}$ (or $e \in \partial \Omega_{h}$ ). We associate with $e$ a unit normal vector $\mathbf{n}_{e}$ directed from $E_{a}$ to $E_{b}(a>b)$. Then, we define the jump and average of a function $f$ on $e$ as

$$
[f]=\left.\left(\left.f\right|_{E_{a}}\right)\right|_{e}-\left.\left(\left.f\right|_{E_{b}}\right)\right|_{e}, \quad\{f\}=\frac{1}{2}\left(\left.\left(\left.f\right|_{E_{a}}\right)\right|_{e}+\left.\left(\left.f\right|_{E_{b}}\right)\right|_{e}\right) .
$$

If $e \in \partial \Omega_{h}$, the above jump and average of $f$ on $e$ reduce to $[f]=\{f\}=\left.\left(\left.f\right|_{E}\right)\right|_{e}$, and the normal vector $\mathbf{n}_{e}$ coincides with the outward normal $\mathbf{n}$.

Given an integer $m \geq 0$, the discontinuous finite element space is

$$
\mathcal{D}_{m}=\left\{\psi \in L^{2}(\Omega) ;\left.\psi\right|_{E} \in \mathcal{P}_{m}(E), \forall E \in \Omega_{h}\right\},
$$

where $\mathcal{P}_{m}(E)$ is the space of polynomials of maximum degree $m$. Let us denote by $p_{w}^{n}$ and $s_{w}^{n}$ the approximation of $p_{w}$ and $s_{w}$ at the $n^{t h}$ time step, respectively, and $\Delta t$ the time step size. To simplify the derivations, we ignore the effect of gravity $(g=0)$. Denote by $(\cdot, \cdot)_{E}$ the $L^{2}(E)$-inner product and by $\langle\cdot, \cdot\rangle_{e}$ the $L^{2}(e)$-inner product. Then, the fully implicit DG discretization of the coupled equations (2.5) and (2.6) is described as follows: Given $\left(p_{w}^{n}, s_{w}^{n}\right) \in \mathcal{D}_{m_{p}} \times \mathcal{D}_{m_{s}}$, find $\left(p_{w}^{n+1}, s_{w}^{n+1}\right) \in \mathcal{D}_{m_{p}} \times \mathcal{D}_{m_{s}}$, such that for $\forall\left(\psi_{p}, \psi_{s}\right) \in \mathcal{D}_{m_{p}} \times \mathcal{D}_{m_{s}}$,

$$
\begin{aligned}
& F_{p}\left(p_{w}^{n+1}, s_{w}^{n+1}\right)=0, \\
& F_{s}\left(p_{w}^{n+1}, s_{w}^{n+1}\right)=0,
\end{aligned}
$$

where

$$
\begin{aligned}
& F_{p}\left(p_{w}^{n+1}, s_{w}^{n+1}\right)=B_{p}+F_{p}^{1}+F_{p}^{2}+F_{p}^{3} \\
& F_{s}\left(p_{w}^{n+1}, s_{w}^{n+1}\right)=B_{s}+F_{s}^{1}+F_{s}^{2}+F_{s}^{3} .
\end{aligned}
$$

In (2.12)-(2.13), $B_{p}$ and $B_{s}$ are bulk integrals obtained from integration by parts:

$$
\begin{aligned}
& B_{p}=\sum_{E \in \Omega_{h}}\left(\frac{\phi}{\Delta t}\left(s_{w}^{n}-s_{w}^{n+1}\right)-q_{n}, \psi_{p}\right)_{E}+\sum_{E \in \Omega_{h}}\left(\lambda_{n}\left(s_{w}^{n+1}\right) \mathbf{K} \nabla\left(p_{w}^{n+1}+p_{c}\left(s_{w}^{n+1}\right)\right), \nabla \psi_{p}\right)_{E}, \\
& B_{s}=\sum_{E \in \Omega_{h}}\left(\frac{\phi}{\Delta t}\left(s_{w}^{n+1}-s_{w}^{n}\right)-q_{w}, \psi_{s}\right)_{E}+\sum_{E \in \Omega_{h}}\left(\lambda_{w}\left(s_{w}^{n+1}\right) \mathbf{K} \nabla p_{w}^{n+1}, \nabla \psi_{s}\right)_{E} .
\end{aligned}
$$

$F_{p}^{1}$ and $F_{s}^{1}$ are jump terms corresponding to face integrals obtained by using the regularity of the exact solution and the boundary conditions [16]:

$$
\begin{aligned}
F_{p}^{1} & =\sum_{e \in \Gamma_{h} \backslash\left(\Gamma_{i n} \cup \Gamma_{0}\right)}\left\langle-\left(\lambda_{n}\left(s_{w}^{n+1}\right) \mathbf{K}\right)^{\mathrm{up}}\left\{\nabla p_{w}^{n+1} \cdot \mathbf{n}_{e}\right\},\left[\psi_{p}\right]\right\rangle_{e}+\sum_{e \in \Gamma_{i n}}\left\langle f_{n}^{i n}, \psi_{p}\right\rangle_{e} \\
& +\sum_{e \in \Gamma_{h} \backslash\left(\Gamma_{i n} \cup \Gamma_{o u t} \cup \Gamma_{0}\right)}\left\langle-\left(\lambda_{n}\left(s_{w}^{n+1}\right) \mathbf{K}\right)^{\mathrm{up}}\left\{\nabla p_{c}\left(s_{w}^{n+1}\right) \cdot \mathbf{n}_{e}\right\},\left[\psi_{p}\right]\right\rangle_{e}, \\
F_{s}^{1} & =\sum_{e \in \Gamma_{h} \backslash\left(\Gamma_{i n} \cup \Gamma_{0}\right)}\left\langle-\left(\lambda_{w}\left(s_{w}^{n+1}\right) \mathbf{K}\right)^{\mathrm{up}}\left\{\nabla p_{w}^{n+1} \cdot \mathbf{n}_{e}\right\},\left[\psi_{s}\right]\right\rangle_{e}+\sum_{e \in \Gamma_{i n}}\left\langle f_{w}^{i n}, \psi_{s}\right\rangle_{e} .
\end{aligned}
$$

$F_{p}^{2}$ and $F_{s}^{2}$ are additional terms for the purpose of stabilization; they vanish for the 
exact solution:

$$
\begin{aligned}
F_{p}^{2} & =\sum_{e \in \Gamma_{h} \backslash\left(\Gamma_{i n} \cup \Gamma_{0}\right)}\left\langle\left(\lambda_{n}\left(s_{w}^{n+1}\right) \mathbf{K}\right)^{\mathrm{up}}\left\{\nabla \psi_{p} \cdot \mathbf{n}_{e}\right\},\left[p_{w}^{n+1}\right]\right\rangle_{e}-\sum_{e \in \Gamma_{\text {out }}}\left\langle\lambda_{n}\left(s_{w}^{n+1}\right) \mathbf{K} \nabla \psi_{p} \cdot \mathbf{n}_{e}, p_{w}^{\text {out }}\right\rangle_{e} \\
& +\sum_{e \in \Gamma_{h} \backslash\left(\Gamma_{i n} \cup \Gamma_{\text {out }} \cup \Gamma_{0}\right)}\left\langle\left(\lambda_{n}\left(s_{w}^{n+1}\right) \mathbf{K}\right)^{\mathrm{up}}\left\{\nabla \psi_{p} \cdot \mathbf{n}_{e}\right\},\left[p_{c}\left(s_{w}^{n+1}\right)\right]\right\rangle_{e}, \\
F_{s}^{2} & =\sum_{e \in \Gamma_{h} \backslash\left(\Gamma_{i n} \cup \Gamma_{0}\right)}\left\langle\left(\lambda_{w}\left(s_{w}^{n+1}\right) \mathbf{K}\right)^{\mathrm{up}}\left\{\nabla \psi_{s} \cdot \mathbf{n}_{e}\right\},\left[p_{w}^{n+1}\right]\right\rangle_{e}-\sum_{e \in \Gamma_{\text {out }}}\left\langle\lambda_{w}\left(s_{w}^{n+1}\right) \mathbf{K} \nabla \psi_{s} \cdot \mathbf{n}_{e}, p_{w}^{\text {out }}\right\rangle_{e}
\end{aligned}
$$

Lastly, $F_{p}^{3}$ and $F_{s}^{3}$ are penalty terms used to constrain the weak continuity of the pressure:

$$
\begin{aligned}
F_{p}^{3} & =\sum_{e \in \Gamma_{h} \backslash\left(\Gamma_{i n} \cup \Gamma_{0}\right)} \gamma\left\|\left(\lambda_{n}\left(s_{w}^{n+1}\right) \mathbf{K}\right)^{\mathrm{up}}\right\|_{\infty}\left\langle\left[p_{w}^{n+1}\right],\left[\psi_{p}\right]\right\rangle_{e}-\sum_{e \in \Gamma_{\text {out }}} \gamma\left\|\left(\lambda_{n}\left(s_{w}^{n+1}\right) \mathbf{K}\right)^{\mathrm{up}}\right\|_{\infty}\left\langle p_{w}^{\text {out }}, \psi_{p}\right\rangle_{e} \\
& +\sum_{e \in \Gamma_{h} \backslash\left(\Gamma_{i n} \cup \Gamma_{\text {out }} \cup \Gamma_{0}\right)} \gamma\left\|\left(\lambda_{n}\left(s_{w}^{n+1}\right) \mathbf{K}\right)^{\mathrm{up}}\right\|_{\infty}\left\langle\left[p_{c}\left(s_{w}^{n+1}\right)\right],\left[\psi_{p}\right]\right\rangle_{e}, \\
F_{s}^{3} & =\sum_{e \in \Gamma_{h} \backslash\left(\Gamma_{i n} \cup \Gamma_{0}\right)} \gamma\left\|\left(\lambda_{w}\left(s_{w}^{n+1}\right) \mathbf{K}\right)^{\mathrm{up}}\right\|_{\infty}\left\langle\left[p_{w}^{n+1}\right],\left[\psi_{s}\right]\right\rangle_{e}-\sum_{e \in \Gamma_{\text {out }}} \gamma\left\|\left(\lambda_{w}\left(s_{w}^{n+1}\right) \mathbf{K}\right)^{\mathrm{up}}\right\|_{\infty}\left\langle p_{w}^{\text {out }}, \psi_{s}\right\rangle_{e}
\end{aligned}
$$

Here, $(\cdot)^{\text {up }}$ means that the quantities are upwinded. The penalty factor $\gamma$ is an important parameter for the performance of the method. We consider the definition in [3] that accounts for the space dimension $d$, polynomial degree $\tilde{m}=\min \left(m_{p}, m_{s}\right)$, and the element size, as follows:

$$
\gamma= \begin{cases}\sigma \frac{\tilde{m}(\tilde{m}+d-1)|e|}{\min \left(\left|E_{a}\right|,\left|E_{b}\right|\right)}, & \text { on } \quad \Gamma_{h} \backslash\left(\Gamma_{\text {in }} \cup \Gamma_{\text {out }} \cup \Gamma_{0}\right), \\ \sigma \frac{\tilde{m}(\tilde{m}+d-1)|e|}{|E|}, & \text { on } \quad \Gamma_{h} \cap \Gamma_{\text {out }},\end{cases}
$$

where $\sigma$ is a user-defined parameter. For general problems with heterogenous porous media, $\gamma$ is effectively scaled by the magnitude of the permeability. In this work, we adopt $\left\|\left(\lambda_{\alpha}\left(s_{w}^{n+1}\right) \mathbf{K}\right)^{\text {up }}\right\|_{\infty}(\alpha=w, n)$ for scaling $\gamma$ as suggested in [17].

The fully implicit DG discretization results in a nonlinear algebraic system

$$
F(X)=0
$$

to be solved at each time step, where $X$ is the vector of unknowns. We note that $F$ is a highly nonlinear function, where the nonlinearities come from the relative permeability $k_{r \alpha}\left(s_{w}\right)$ and the capillary pressure function $p_{c}\left(s_{w}\right)$. Extra difficulties for solving (2.14) are induced by the heterogeneity of $\phi$ and $K$.

3. Nonlinearly preconditioned inexact Newton algorithms. In this section, we describe a class of nonlinearly preconditioned inexact Newton algorithms for solving (2.14). We first recall the inexact Newton method with backtracking (INB) $[14,15]$ as the outer iterative process: take the solution of the previous time step or the initial condition as the initial guess $X_{0}=X^{n}$, then the next approximate solution $X_{k+1}$ is obtained by

$$
X_{k+1}=X_{k}+\lambda_{k} S_{k}, \quad k=0,1, \ldots
$$

where the inexact Newton direction $S_{k}$ satisfies

$$
\left\|J_{k} S_{k}+F\left(X_{k}\right)\right\| \leq \eta_{k}\left\|F\left(X_{k}\right)\right\| .
$$


Here $J_{k}=F^{\prime}\left(X_{k}\right)$ is the Jacobian matrix. The step length $\lambda_{k} \in[0,1]$ is determined by a line search procedure [14]. $\eta_{k} \in[0,1)$ is a forcing term that determines how accurately the Jacobian system needs to be solved. To enhance the robustness of INB, $\eta_{k}$ can be computed based on norms that are by-products of the iteration, as suggested by Eisenstat and Walker [15]. The nonlinear iteration is stopped if

$$
\left\|F\left(X_{k}\right)\right\| \leq \gamma_{r}\left\|F\left(X_{0}\right)\right\|,
$$

where $\gamma_{r}$ is a prescribed relative tolerance for the nonlinear solver.

Slow convergence of INB occurs when the value of $\lambda_{k}$ is small. To accelerate the convergence, nonlinear preconditioning is introduced to balance the overall nonlinearities of the system so that a single search direction $S_{k}$ can benefit all components of the residual function $F$.

The key idea of nonlinear elimination (NE) preconditioning is to first identify part of $F$ responsible for the slow convergence and then approximately eliminate it using an inner iteration. We partition the components of $F$ into 'good' and 'bad' groups labeled with superscripts 'g' and 'b':

$$
F(X)=\left[\begin{array}{c}
F^{b}\left(X^{b}, X^{g}\right) \\
F^{g}\left(X^{b}, X^{g}\right)
\end{array}\right],
$$

and $X=\left(X^{b}, X^{g}\right)$. Similar to Gaussian elimination for linear problems, we need a nonsingular block as the pivot. Here we assume that $\frac{\partial F^{b}}{\partial X^{b}}$ is nonsingular, for any $X^{g}$ in a projection set $\left\{X^{g} \mid\left(X^{b}, X^{g}\right) \in \mathbb{R}^{N}\right\}$. Based on the implicit function theorem of calculus, there exists a function $g\left(X^{g}\right)$ such that

$$
F^{b}\left(g\left(X^{g}\right), X^{g}\right)=0 .
$$

We call $G(X)=\left(g\left(X^{g}\right), X^{g}\right)$ as the cut-extension of $X$, that keeps the good part of $\mathrm{X}$ and replaces the bad part of $\mathrm{X}$ by a subspace vector that satisfies a subset of the nonlinear system. The modified system reads as follows: Find $Y=G(X)$ such that

$$
F(Y)=\left[\begin{array}{c}
F^{b}\left(g\left(X^{g}\right), X^{g}\right) \\
F^{g}\left(g\left(X^{g}\right), X^{g}\right)
\end{array}\right]=\left[\begin{array}{c}
0 \\
F^{g}\left(g\left(X^{g}\right), X^{g}\right)
\end{array}\right]=0
$$

which will be solved by INB described in the beginning of the section. (3.6) is often written as

$$
F(G(X))=0,
$$

and is called a right-preconditioned nonlinear system. By using the theory of affine invariance, Gong and Cai [18] discussed some criteria for choosing the to-be-eliminated components under certain conditions so that NE improves the convergence of Newton iterations. We refer to the details in the reference.

During the outer INB process, the relative reduction of the residual

$$
\rho_{k}=\frac{\left\|F\left(X_{k}\right)\right\|}{\left\|F\left(X_{k-1}\right)\right\|}
$$

can be used to measure the effectiveness of the $k^{\text {th }}$ Newton solution $X_{k}$. If $\rho_{k}$ is too large (i.e. $\rho_{k}>\rho_{0}$, where $0<\rho_{0}<1$ is a preselected parameter), the NE preconditioner is then introduced to produce a different Newton solution $X_{k}^{*}$. On 
the other hand, if $\rho_{k}$ is small, then we proceed with the classical INB without any nonlinear preconditioning for this Newton step. In the NE preconditioner, we solve the following nonlinear system

$$
\mathcal{F}_{k}(X) \equiv\left[\begin{array}{c}
F^{b}\left(X^{b}, X^{g}\right) \\
X^{g}-X_{k}^{g}
\end{array}\right]=0
$$

by using INB with the initial guess $X_{k} . X_{k}^{*}$ is accepted as the approximate solution if the stopping condition $\left\|\mathcal{F}_{k}\left(X_{k}^{*}\right)\right\| \leq \gamma_{r}^{\mathrm{NE}}\left\|\mathcal{F}_{k}\left(X_{k}\right)\right\|$ is satisfied, where $\gamma_{r}^{\mathrm{NE}}$ is the relative tolerance for the nonlinear solver. Here the 'inverse' of $\mathcal{F}_{k}(X)=0$ is considered as a nonlinear preconditioner of $F$, that is, $X_{k}^{*}=G\left(X_{k}\right)$.

In the modified nonlinear system (3.9), $F^{b}$ is simply a restriction of $F$ to a subset of equations, while the replacement for the good components $X^{g}-X_{k}^{g}=0$ is trivial, which results in an identity part of the inner Jacobian. Therefore, the modified nonlinear system is often better conditioned. In addition, the modified nonlinear system does not need to be solved exactly; a relatively large tolerance (i.e., $\gamma_{r}^{\mathrm{NE}}=$ $10^{-1}$ ) is sufficient for the inner Newton to return an updated solution that results in more balanced nonlinearities. Hence, solving (3.9) is often easier than solving the original problem.

A high level description of the INB-NE algorithm for solving (2.14) at each time step is presented in Algorithm 3.1.

Algorithm 3.1 The nonlinear elimination preconditioned inexact Newton algorithm with backtracking (INB-NE): given absolute and relative nonlinearity bounds $\varepsilon$ and

$\rho_{0}$

Step 1 Take the solution of the previous time step or the initial condition as the initial guess $X_{0}=X^{n}$. Set $k=0, X_{-1}=X_{0}$.

Step 2 Check convergence:

- If the global stopping condition $\left\|F\left(X_{k}\right)\right\| \leq \gamma_{r}\left\|F\left(X_{0}\right)\right\|$ is satisfied, stop and update $X^{n+1}=X_{k}$.

- If $\left\|F\left(X_{k}\right)\right\| \geq \varepsilon$ and $\left\|F\left(X_{k}\right)\right\| /\left\|F\left(X_{k-1}\right)\right\| \geq \rho_{0}$, go to Step 3; otherwise, go to Step 4.

Step 3 The NE step: perform the subspace correction:

- Identify the to-be-eliminated components.

- Form and evaluate the nonlinear function $\mathcal{F}_{k}(X)$ in (3.9).

- Approximately solve $\mathcal{F}_{k}(X)=0$ using the classical INB with initial guess $X_{k}$.

- Take the solution of the inner Newton iteration as $X_{k}^{*}$ and update $X_{k}=$ $X_{k}^{*}$.

Step 4 The global INB step:

- Analytically construct the global Jacobian matrix $J_{k}=F^{\prime}\left(X_{k}\right)$.

- Inexactly solve $J_{k} S_{k}=-F\left(X_{k}\right)$.

- Compute $\lambda_{k}$ using the cubic backtracking line search.

- Update $X_{k+1}=X_{k}+\lambda_{k} S_{k}$.

- Set $k=k+1$, go to Step 2 .

In Step 2, if the residual norm $\left\|F\left(X_{k}\right)\right\|$ is less than the given value of $\varepsilon$, the intermediate solution is considered to be close to the desired one, therefore, the NE step can be skipped in order to save the overhead of the nonlinear preconditioning.

In Step 3, a subspace Newton method is performed to remove subspace high nonlinearities before applying a global nonlinear update, so it is essential to effectively 
identify the bad components to be eliminated in the nonlinear system. Below we first introduce the Saturation component-wise approach proposed in [50] as a base to discuss how to construct the subspace nonlinear function $\mathcal{F}_{k}(X)$.

3.1. A strategy based on the pointwise value of saturation. This approach is based on a hybrid physical-algebraic partition of the residual function, where the bad components are selected only from the saturation field whose residual values often dominate those from the pressure field. We first introduce some notations. Let $\mathcal{S}=\left\{s_{1}, s_{2}, \ldots, s_{N_{s}}\right\}$ be an index set with respect to the saturation components, where each index corresponds to an unknown component $X_{k, s_{i}}$ and a nonlinear function $F_{s_{i}}$, and $N_{s}$ is the number of elements of $\mathcal{S}$. We note that $\mathcal{S}$ is a subset of the index set $\mathcal{N}=\{1, \ldots, N\}$. At the $k^{\text {th }}$ Newton iteration, the index set is decomposed into two parts $\mathcal{S}_{k}^{b}$ and $\mathcal{S}_{k}^{g}$, such that $\mathcal{S}_{k}^{g}=\mathcal{S} \backslash \mathcal{S}_{k}^{b}$, where $\mathcal{S}_{k}^{b}$ and $\mathcal{S}_{k}^{g}$ correspond to the components that have strong and weak nonlinearities, respectively. Let $F_{\mathcal{S}} \in \mathbb{R}^{N_{s}}$ be the vector of residual function with respect to the saturation components (i.e., the wetting phase part of $F$ ), then the bad subset $\mathcal{S}_{k}^{b}$ is determined as follows,

$$
\mathcal{S}_{k}^{b}=\left\{s_{i} \mid \text { If }\left|F_{s_{i}}\left(X_{k}\right)\right|>\varrho\left\|F_{\mathcal{S}}\left(X_{k}\right)\right\|_{\infty}, i=1, \ldots, N_{s}, s_{i} \in S\right\},
$$

where $\varrho$ is a preselected parameter. With this partition, one can define two subspaces

$$
\begin{aligned}
& \mathcal{V}_{k}^{b}=\left\{V \mid V=\left(V_{1}, \ldots, V_{N}\right)^{T} \in \mathbb{R}^{N}, V_{n}=0 \text { if } n \notin \mathcal{S}_{k}^{b}, n=1, \ldots, N\right\}, \\
& \mathcal{V}_{k}^{g}=\left\{V \mid V=\left(V_{1}, \ldots, V_{N}\right)^{T} \in \mathbb{R}^{N}, V_{n}=0 \text { if } n \in \mathcal{S}_{k}^{b}, n=1, \ldots, N\right\},
\end{aligned}
$$

respectively. The corresponding restriction operators are denoted as $\mathcal{R}_{k}^{b}$ and $\mathcal{R}_{k}^{g}$, that are sub-identity matrices mapping the vectors from $\mathbb{R}^{N}$ to $\mathcal{V}_{k}^{b}$ and $\mathcal{V}_{k}^{g}$, respectively. Then, the subspace nonlinear function in the NE step is defined as

$$
\mathcal{F}_{k}(X) \equiv \mathcal{R}_{k}^{b}(F(X))+\mathcal{R}_{k}^{g}\left(X-X_{k}\right) .
$$

Remark 3.1. The saturation component-wise approach is adaptive in the sense that the subspace nonlinear system $\mathcal{F}_{k}(X)=0$ may be different for different time steps.

In this approach, only the dominant residual components of the saturation field are identified and eliminated. In practice, such a single-field elimination strategy may cause stagnation or even failure for the inner Newton iteration when the subspace system involves locally stiff coefficients or highly nonlinear capillary effect. For such cases, the difficulty is often caused by the strongly coupled physical phenomena arising from the interaction between the pressure and the saturation in a heterogeneous media of high contrast. To overcome this difficulty, we propose two new elimination strategies, respectively, a multiplicative field-split approach and a coupled element-block approach.

3.2. A strategy based on the field-splitting of pressure and saturation. This strategy is a right version of left multiplicative nonlinear preconditioning, namely, the multiplicative Schwarz preconditioned inexact Newton (MSPIN) method $[33,35]$, where the subproblems with respect to different physical variables are solved sequentially, similar to a Gauss-Seidel iteration. In the NE context, we perform the subspace correction step (Step 3 in Algorithm 3) in two stages to eliminate alternatively the pressure components and the saturation components. We call this strategy 
as a multiplicative field-split approach. Specifically, at the first stage, we define two subspaces

$$
\begin{aligned}
& \mathcal{V}_{1}^{b}=\left\{V \mid V=\left(V_{1}, \ldots, V_{N}\right)^{T} \in \mathbb{R}^{N}, V_{n}=0 \text { if } n \in \mathcal{S}, n=1, \ldots, N\right\}, \\
& \mathcal{V}_{1}^{g}=\left\{V \mid V=\left(V_{1}, \ldots, V_{N}\right)^{T} \in \mathbb{R}^{N}, V_{n}=0 \text { if } n \notin \mathcal{S}, n=1, \ldots, N\right\} .
\end{aligned}
$$

The corresponding restriction operators are denoted as $\mathcal{R}_{1}^{b}$ and $\mathcal{R}_{1}^{g}$, respectively. Then, we solve the following subspace nonlinear system with the initial guess $X_{k}$,

$$
\mathcal{F}_{1}(X) \equiv \mathcal{R}_{1}^{b}(F(X))+\mathcal{R}_{1}^{g}\left(X-X_{k}\right)=0 .
$$

We denote $\tilde{X}_{k}$ as the approximate solution for the first stage. At the second stage, we define two counterpart subspaces

$$
\begin{aligned}
& \mathcal{V}_{2}^{b}=\left\{V \mid V=\left(V_{1}, \ldots, V_{N}\right)^{T} \in \mathbb{R}^{N}, V_{n}=0 \text { if } n \notin \mathcal{S}, n=1, \ldots, N\right\}, \\
& \mathcal{V}_{2}^{g}=\left\{V \mid V=\left(V_{1}, \ldots, V_{N}\right)^{T} \in \mathbb{R}^{N}, V_{n}=0 \text { if } n \in \mathcal{S}, n=1, \ldots, N\right\} .
\end{aligned}
$$

The corresponding restriction operators are denoted as $\mathcal{R}_{2}^{b}$ and $\mathcal{R}_{2}^{g}$, respectively. Then, we solve the following subspace nonlinear system with the initial guess $\tilde{X}_{k}$,

$$
\mathcal{F}_{2}(X) \equiv \mathcal{R}_{2}^{b}(F(X))+\mathcal{R}_{2}^{g}\left(X-\tilde{X}_{k}\right)=0 .
$$

Finally, we take the approximate solution of (3.13) as $X_{k}^{*}$.

Remark 3.2. Denote $G_{1}(X)$ and $G_{2}(X)$ as the 'inverses' of (3.12) and (3.13) respectively, then, we have the composite formulation of the right nonlinear preconditioner

$$
X_{k}^{*}=G\left(X_{k}\right)=G_{2}\left(G_{1}\left(X_{k}\right)\right) .
$$

Remark 3.3. The multiplicative field-split approach is static in the sense that the selection of bad components does not change with $k$.

Remark 3.4. A widely used linear two-stage preconditioner in the community of reservoir simulation, the constrained pressure residual (CPR) method, is based on subblocks of the Jacobian matrix and uses an approximate pressure solve such as algebraic multigrid (AMG) to constrain the residual of the full system [19, 34]. Compared to CPR-AMG, the multiplicative field-split approach does not require derivation or assembly of an elliptic pressure equation to make use of AMG. It is applied directly to the nonlinear system to reduce the nonlinear stiffness.

In general, it may be time consuming to eliminate alternatively all components of different physical fields per NE application. One variant of the multiplicative fieldsplit approach is to identify and eliminate only the dominant residual components of each field using an algebraic criteria similar to (3.10). However, in our numerical experiments, we observe that the full field approach is efficient for the problems concerned in this paper.

3.3. A strategy based on the field-coupling of pressure and saturation. The strong nonlinearities of the system are often related to certain critical features (e.g., discontinuity of permeability coefficients, spatial variation of capillary effects, 
complex source terms, and corner/fault/void singularities) that appear in certain local regions. In such a situation, a small number of components defined in the local regions, or nearby elements, may contribute to a large percentage of $\|F\|$. On the other hand, the DG finite element discretization used in this work leads to a tight coupling of the unknown variables within an element. Based on these two observations, we devise a coupled element-block approach, that is, when one component defined on a particular element is selected to be eliminated, all other components associated with this element are also eliminated.

Specifically, let $n_{\psi_{p}}$ and $n_{\psi_{s}}$ be the numbers of basis functions for the pressure and saturation in element $E$, respectively. Then, each element has totally $n_{\psi}=n_{\psi_{p}}+n_{\psi_{s}}$ degrees of freedom with respect to the two variables. We denote $\left\{E_{c}: c=1, \ldots, n_{\psi}\right\}$ as the index set that collects the indices corresponding to the degrees of freedom defined on element $E$ (i.e., $n_{\psi}$ unknown components $X_{k, E_{c}}$ and $n_{\psi}$ nonlinear residual components $\left.F_{E_{c}}\right)$. At the $k^{\text {th }}$ Newton iteration, we decompose $\Omega_{h}$ into a 'bad' subset $\mathcal{E}_{k}^{b}$ and a 'good' subset $\mathcal{E}_{k}^{g}$, such that $\mathcal{E}_{k}^{g}=\Omega_{h} \backslash \mathcal{E}_{k}^{b}$. The bad subset $\mathcal{E}_{k}^{b}$ is defined as

$$
\mathcal{E}_{k}^{b}=\left\{E \mid \text { If } \max _{c}\left\{\left|F_{E_{c}}\left(X_{k}\right)\right|\right\}>\varrho\left\|F\left(X_{k}\right)\right\|_{\infty}, c=1, \ldots, n_{\psi}, E \in \Omega_{h}\right\} .
$$

For the DG finite element discretization in space, the calculation of $F$ involves the unknowns and derivatives from two neighboring elements, thus new jumps may be produced in the residual function across the interface between the good and bad regions $[8,48,51]$. To avoid such jumps, we extend $\mathcal{E}_{k}^{b}$ to a larger subset $\mathcal{E}_{k}^{b, \delta}$ by including $\delta_{n}$ layers of neighboring elements so that the interface is far away from the local high nonlinearities. An example for illustration of the bad region on a $2 \mathrm{D}$ mesh is shown in Figure 1. With the subset $\mathcal{E}_{k}^{b, \delta}$, we define two subspaces

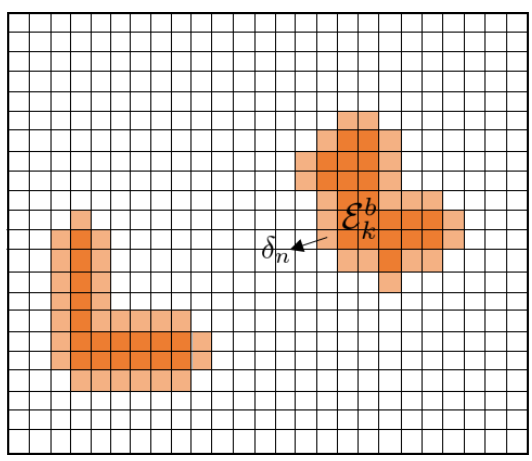

FIG. 1. An example for illustration of the bad region on a $2 D$ mesh.

$$
\begin{aligned}
& \mathcal{V}_{k}^{b, \delta}=\left\{V \mid V=\left(V_{1}, \ldots, V_{N}\right)^{T} \in \mathbb{R}^{N}, V_{E_{c}}=0 \text { if } E \notin \mathcal{E}_{k}^{b, \delta}, c=1, \ldots, n_{\psi}\right\} \\
& \mathcal{V}_{k}^{g, \delta}=\left\{V \mid V=\left(V_{1}, \ldots, V_{N}\right)^{T} \in \mathbb{R}^{N}, V_{E_{c}}=0 \text { if } E \in \mathcal{E}_{k}^{b, \delta}, c=1, \ldots, n_{\psi}\right\} .
\end{aligned}
$$

The corresponding restriction operators are denoted as $\mathcal{R}_{k}^{b}$ and $\mathcal{R}_{k}^{g}$, respectively. Then, the subspace nonlinear function $\mathcal{F}_{k}(X)$ in the NE step can be defined the same as (3.11).

The coupled element-block approach is based on a decomposition of the mesh $\Omega_{h}$, which can be viewed as an extension of the point-wise approach $[22,51]$ that has been used successfully for multi-component systems. However, the point-wise approach is 
not suitable for our work since we use mixed order polynomials for different variables in the DG scheme. On the other hand, the coupled element-block approach generalizes the saturation component-wise approach [50] by including the continuous subdomain (the associated element and its neighbors) to the bad subset where the change of the physical variables may be abrupt. A performance comparison of these approaches is presented in the numerical experiments.

3.4. Linear solver. A linear solver is required to solve the Jacobian systems arising from both the global Newton iteration and the nonlinear elimination process. Good candidates include the class of Krylov subspace methods with effective linear preconditioners. In this study, we use a restricted additive Schwarz (RAS) [6] preconditioned generalized minimal residual (GMRES) method [43] to solve the Jacobian systems.

We rewrite the Jacobian system in the following general form

$$
J M^{-1} y=b, \quad \text { with } \quad x=M^{-1} y,
$$

where $J$ is the Jacobian matrix, $M$ is the preconditioner, $x$ is the solution, and $b$ is the right-hand side. Denote by $n p$ the number of processor cores of the parallel computer, we partition the finite element mesh $\Omega_{h}$ into $n p$ nonoverlapping subdomains $\Omega_{l}$ (i.e. $\Omega_{i} \cap \Omega_{j}=\emptyset, \forall i \neq j$ ) for $l=1, \ldots, n p$, such that $\Omega_{h}=\Omega_{1} \cup \cdots \cup \Omega_{n p}$. The subvector associated with $\Omega_{l}$ is denoted as $y_{l}$. We then extend $\Omega_{l}$ to overlap with its neighbors by $\delta_{l}$ layers of mesh elements and denote the overlapping subdomain as $\Omega_{l}^{\delta}$. On each overlapping subdomain, we define the corresponding subvector $y_{l}^{\delta}$ and the restriction operator $R_{l}^{\delta}$ that maps the global vector of unknowns in $\Omega_{h}$ to $y_{l}^{\delta}$, i.e.

$$
y_{l}^{\delta}=R_{l}^{\delta} y=\left(\begin{array}{ll}
I & 0
\end{array}\right)\left(\begin{array}{c}
y_{l}^{\delta} \\
y \backslash y_{l}^{\delta}
\end{array}\right) .
$$

Figure 2 shows a sample partition of a mesh into 8 subdomains (left) and an example of an overlapping subdomain (right). We denote by $R_{l}^{0}$ the restriction operator that
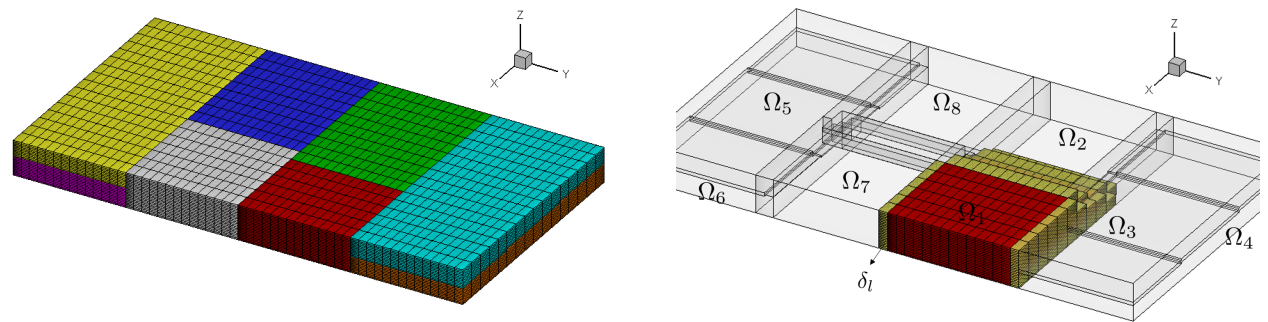

FIG. 2. (left) A sample partition of a mesh into 8 subdomains. (right) A non-overlapping subdomain colored in red and one layer of overlap colored in yellow.

returns $y_{l}$ defined on the nonoverlapping subdomain. Then, the RAS preconditioner $[6]$ is defined as

$$
M_{\mathrm{RAS}}^{-1}=\sum_{l=1}^{n p}\left(R_{l}^{0}\right)^{T}\left(J_{l}\right)^{-1} R_{l}^{\delta} .
$$

In (3.16), $\left(J_{l}\right)^{-1}$ is understood as an approximate inverse of the subdomain Jacobian matrix, its product with a vector is computed by solving a subdomain linear system inexactly using an incomplete LU (ILU) factorization of $J_{l}$. 
Remark 3.5. The Jacobian matrix $J$ is a key component in Newton-type methods. In this study, we choose to analytically compute $J$ using the chain rule since the exact Jacobian matrix brings added robustness. We refer to [38] for more details for the construction of $J$.

Remark 3.6. In general, the subspace Jacobian system is better conditioned than the global Jacobian system. For the purpose of efficiency, the subspace Jacobian matrix and the subspace preconditioner do not need to be recomputed for every inner Newton iteration. Later, we show by numerical tests that reusing these subspace operators helps to save the total compute time.

4. Numerical experiments. In this section, we provide some examples to illustrate the robustness and efficiency of the proposed methods. We first validate our discretization scheme and the fully implicit solver using a benchmark problem in a homogeneous porous media. Then, we study the performance of various INB-NE methods for heterogeneous porous media and focus on: (1) the robustness with respect to some physical and numerical parameters; and (2) the comparison of performance between INB and INB-NE.

The algorithms are implemented using libMesh [28] for the finite element assembly, and PETSc [2] for the inexact Newton-Krylov solver. All computations are carried out on the Shaheen2 supercomputer, which has two 16-core Intel Haswell CPUs and $128 \mathrm{~GB}$ local memory in each of its compute nodes. We use piecewise quadratic polynomials for the pressure and piecewise linear polynomials for the saturation: $m_{p}=2$, $m_{s}=1$ [16]. The user-defined penalty parameter in the NIPG scheme is given as $\sigma=10$ for all the tests. We use the following parameters in our solvers if they are not specifically stated. The relative tolerance for the global Newton iteration is set to be $\gamma_{r}=10^{-5}$. The relative tolerance for the inner Newton iteration is $\gamma_{r}^{\mathrm{NE}}=10^{-1}$. The inner Newton iteration is also stopped by a maximum number of iterations 15 . The subspace Jacobian and preconditioner are evaluated only once at the first inner Newton iteration and are then reused for the rest of the computation. The restart value of GMRES is fixed at 100. The size of overlap in the linear RAS preconditioner is fixed to $\delta_{l}=1$. An ILU factorization with 2 fill-in levels is used to solve the subdomain linear systems. In all cases, the effect of gravity is neglected $(g=0)$ for simplicity, and the void of the media is initially fully saturated with oil, i.e., $s_{w}^{0}=0$. Except for the first example, we consider injection and production well sources in the simulation. The wetting-phase fluid is injected with a constant rate at the injector. A Peaceman well model [10] is used for the producer with the well radius $r_{w}$, the skin factor $s_{k}$, and the bottom hole pressure $p_{b h}$ given specifically in the examples.

For the implementation of NE, we perform two steps to assemble the modified nonlinear system (3.9). The first step is to identify the to-be-eliminated components and the counterpart good components. In the second step, we reuse the routines to assemble Jacobians and residuals for the global Newton solver, then replace the equations corresponding to the good components by $\left(X-X_{k}\right)_{n}=0$. Here $(\cdot)_{n}$ means the $n^{\text {th }}$ component of the vector. Then, the solve of $\mathcal{F}_{k}(X)=0$ can be performed in the whole space. The inner Newton solver shares the same framework and the same number of parallel processes with the global solver, thus standard nonlinear solver software packages can be used with some slight modification. This implementation saves the memory as well as the overhead to create inner solvers per outer iteration. The efficiency of the proposed method can be further improved if some dynamic load balance technique is used. One strategy would be to dynamically partition and redistribute the bad subset among all the processor cores so that each core owns 
almost equal number of bad components.

In the rest of this paper, ' $\mathrm{NI}_{g}$ ' denotes the averaged number of global Newton iterations per time step; ' $\mathrm{LI}_{g}$ ' denotes the averaged number of GMRES iterations per global Newton iteration; ' $\mathrm{N}_{\mathrm{NE}}$ ' is the averaged number of subspace correction steps in $\mathrm{NE}$ per time step; ' $\mathrm{NI}_{\mathrm{NE}}$ ' refers to the averaged number of Newton iterations per subspace correction in NE; ' $\mathrm{LI}_{\mathrm{NE}}$ ' is the averaged number of GMRES iterations per Newton iteration in $\mathrm{NE}$; ' $\mathrm{T}_{\mathrm{NE}}$ ' is the compute time in seconds for all $\mathrm{NE}$ applications per time step; and ' $\mathrm{T}_{t}$ ' is the total compute time in seconds per time step. These numbers reported in the tests are obtained by taking average for 10 time steps. We also denote by 'Lag' $(=1,2, \ldots, \infty)$ the recomputed frequency of the subspace Jacobian and preconditioner in the NE step.

4.1. Example 1: The Buckley-Leverett problem. We first consider the Buckley-Leverett problem in a 1D homogeneous porous media, which has well-known analytical solution for cases with different fluid properties and zero capillary pressure [21]. In the test, water (wetting-phase) is injected with a constant flow rate at one end $\left(\Gamma_{i n}\right)$ to displace oil to the other end $\left(\Gamma_{\text {out }}\right)$. The pressure is kept constant at the production end and the capillary pressure is neglected. The absolute permeability tensor is taken as $\mathbf{K}=K \mathbf{I}$, where $\mathbf{I}$ is the identity matrix and $K$ is a positive real number. The relevant data for this problem are provided in Table 1. The mesh size is $100 \times 1 \times 1$. The classical INB is used for the simulation with the time step size $\Delta t=1$ day. The simulations are carried out using 10 processor cores. We compare the results of our fully implicit DG scheme with an IMPES based two-point fluxapproximation (TPFA) scheme offered by the open source code MRST (MATLAB Reservoir Simulation Toolbox [32]). Numerical and analytical solutions of wetting saturation are plotted in Figure 3. It is observed that the profiles of our scheme capture the discontinuities well with less numerical dispersion than the MRST solution, due to its higher order (linear) approximation. Figure 4 shows a convergence test using different mesh sizes for our scheme. We see from the figure that the saturation profiles are stable and converge as the mesh is refined.

TABLE 1

Relevant parameters for Example 1

\begin{tabular}{ll}
\hline Domain dimensions & $300 \mathrm{~m} \times 1 \mathrm{~m} \times 1 \mathrm{~m}$ \\
Rock properties & $\phi=0.2, K=1 \mathrm{mD}$ \\
Fluid properties & $\mu_{w}=2 \mathrm{cP}, \mu_{n}=3 \mathrm{cP}$ \\
& $\rho_{w}=\rho_{n}=1000 \mathrm{~kg} / \mathrm{m}^{3}$ \\
Relative permeabilities & $\beta=2 \mathrm{in}(2.8)$ \\
Capillary pressure & $\bar{B}_{c}=0 \mathrm{in}(2.8)$ \\
Residual saturations & $s_{r w}=0, s_{r n}=0.2$ \\
Injection rate & $5 \times 10^{-4} \mathrm{PV} /$ day \\
\hline
\end{tabular}

4.2. Example 2: A square horizontal domain with obstacles. By this example, we test the INB-NE methods for oil displacement driven by diagonal well sources in a square horizontal domain with specific geometry setup. In the test, an injector is located in the middle of the domain and two producers are located on two diagonal corners, respectively. We design two rectangular obstacles near the left bottom corner that exert an impedance on the flow movement, as shown in Figure 5(a). All boundaries are impermeable $\left(\Gamma_{0}\right)$. An unstructured mesh consisting of 2,436 elements is used for the test, leading to 85,260 degrees of freedom. Other relevant parameters are provided in Table 2. In the heterogeneous media cases, the random 


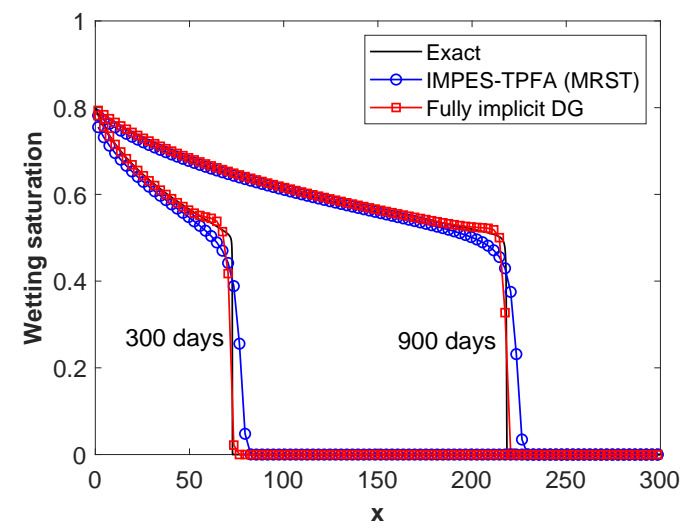

FIG. 3. Wetting saturation of the Buckley-Leverett problem. The mesh size is $100 \times 1 \times 1$.



FIG. 4. Wetting saturation of the Buckley-Leverett problem at 900 days obtained using the fully implicit DG scheme with different mesh sizes.

porosity and permeability fields are generated by MRST. The coupled element-block INB-NE method is used for the simulation, and the preselected parameters are given as $\rho_{0}=0.25, \varepsilon=5 \times 10^{-4}, \varrho=0.05$, and $\delta_{n}=1$. The simulations are carried out using 256 processor cores. Figure 6 shows the wetting saturation at $t=500$ days for cases with different viscosity ratios and different capillary strength in a homogeneous or heterogeneous media. As the simulation moves forward, the injecting fluid pushes the interface to the two diagonal wells correspondingly. The two obstacles significantly change the flow path in the left bottom part of the domain. In case (b), the combination of viscosity differences and permeability heterogeneity introduces the viscous fingering effects [32]. For cases (c)-(d), there is a significant effect of the capillary pressure function that results in a more diffusive solution.

For cases with high contrast of permeability heterogeneity and capillary effect, the classical INB may diverge due to the failure of line search. We present a comparison of INB and the proposed INB-NE methods for the heterogeneous media cases in Table 3. The saturation component-wise approach [50] is also examined in the test where the prescribed parameter $\varrho$ in (3.10) is selected for optimal performance. The time step size is $\Delta t=1$ day for all cases. For cases with zero capillary pressure function 


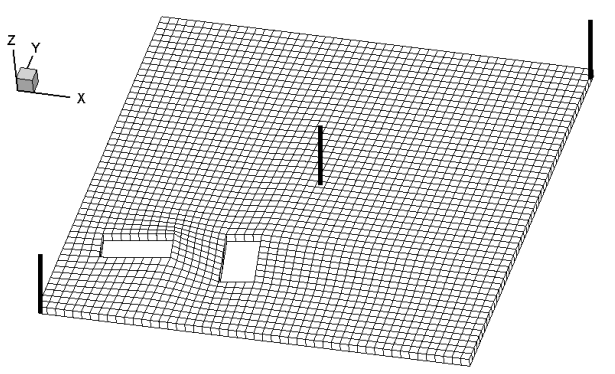

(a) Unstructured mesh with 2,436 elements

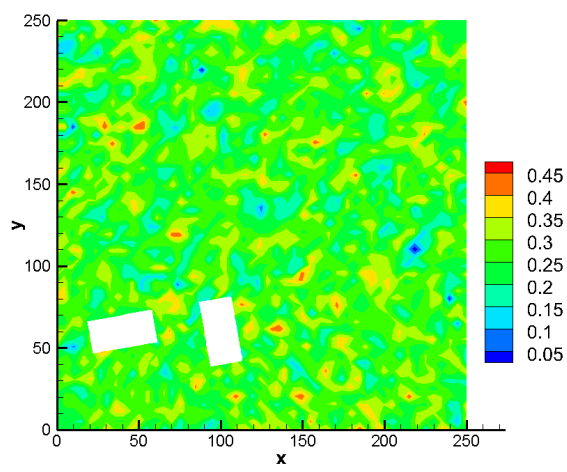

(b) Porosity

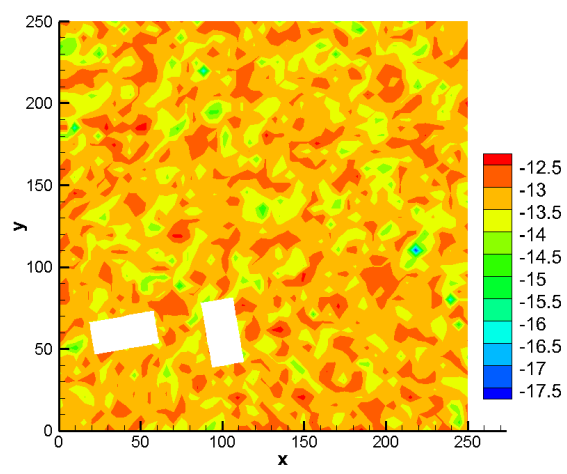

(c) Permeability $\left(\log _{10} K\right)$

FIG. 5. Example 2: a square horizontal domain with obstacles.

TABLE 2

Relevant parameters for Example 2

\begin{tabular}{ll}
\hline Domain dimensions & $250 \mathrm{~m} \times 250 \mathrm{~m} \times 5 \mathrm{~m}$ \\
Rock properties & homogeneous: $\phi=0.2, K=100 \mathrm{mD}$ \\
& heterogeneous: $\phi \in[0.01,0.48], K \in[0.00177,718.69] \mathrm{mD}$ \\
Fluid properties & $\mu_{w}(\mathrm{cP}) / \mu_{n}(\mathrm{cP})=1 / 1,1 / 2,1 / 4$ \\
& $\rho_{w}=1025 \mathrm{~kg} / \mathrm{m}^{3}, \rho_{n}=849 \mathrm{~kg} / \mathrm{m}^{3}$ \\
Relative permeabilities & $\beta=2$ in $(2.8)$ \\
Capillary pressure & $\bar{B}_{c}=B_{c} / \sqrt{K}$ in $(2.8), B_{c}=0-18 \mathrm{bar} \cdot \mathrm{mD}^{1 / 2}$ \\
Residual saturations & $s_{r w}=0, s_{r n}=0$ \\
Injection rate & $43.2 \mathrm{~m}^{3} /$ day \\
Production well & $r_{w}=0.1 \mathrm{~m}, s_{k}=0, p_{b h}=1$ bar $[10]$ \\
\hline
\end{tabular}

$\left(B_{c}=0\right)$, all methods successfully resolve the evolution of the two-phase flow. When the capillary effect is involved, the classical INB diverges in Case 3 and Case 5, while the saturation component-wise INB-NE diverges in Case 5 though a minimum value of $\varrho$ is used and the subspace Jacobian and preconditioner are recomputed every inner Newton iteration. In contrast, the proposed multiplicative field-split INB-NE and coupled element-block INB-NE converge well for all cases, yielding better robustness with respect to the strong capillary effect. Compared to INB, the application of NE significantly reduces the number of global Newton iterations. This saves the total compute time though it pays the price to solve a subspace nonlinear system. From the table we see that the compute times for the NE preconditioner are about $40 \%-60 \%$ 


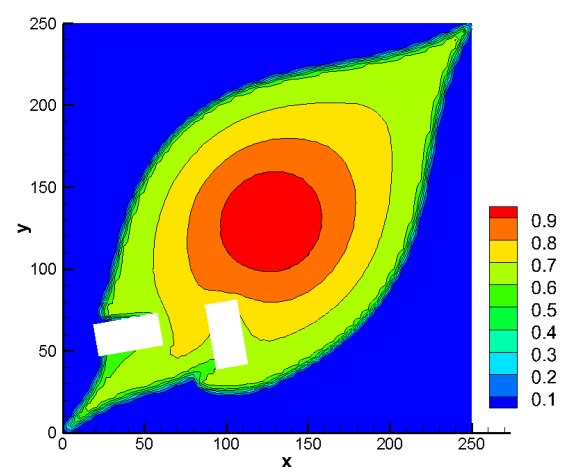

(a) Homogeneous media, $\frac{\mu_{w}}{\mu_{n}}=\frac{1}{2}, B_{c}=0$

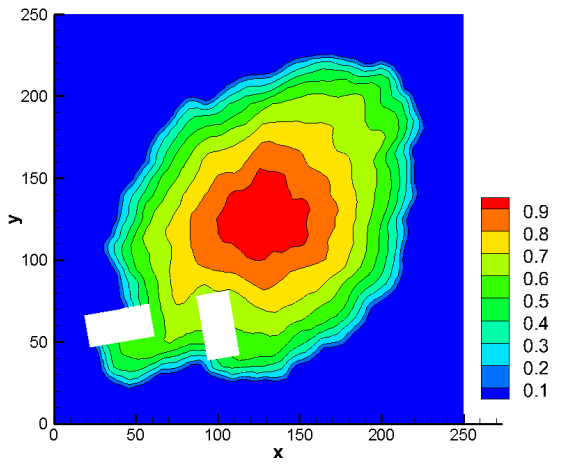

(c) Heterogeneous media, $\frac{\mu_{w}}{\mu_{n}}=\frac{1}{2}, B_{c}=8$

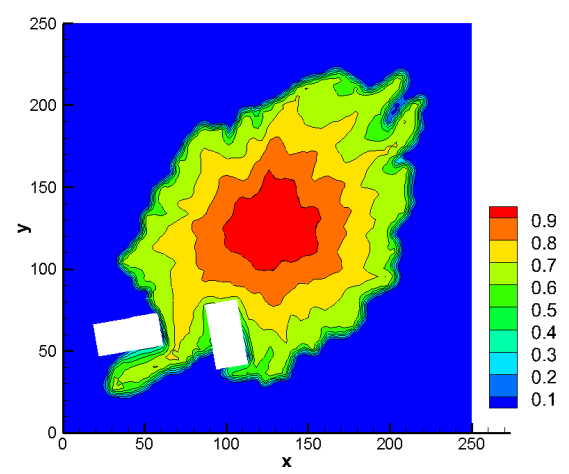

(b) Heterogeneous media, $\frac{\mu_{w}}{\mu_{n}}=\frac{1}{2}, B_{c}=0$

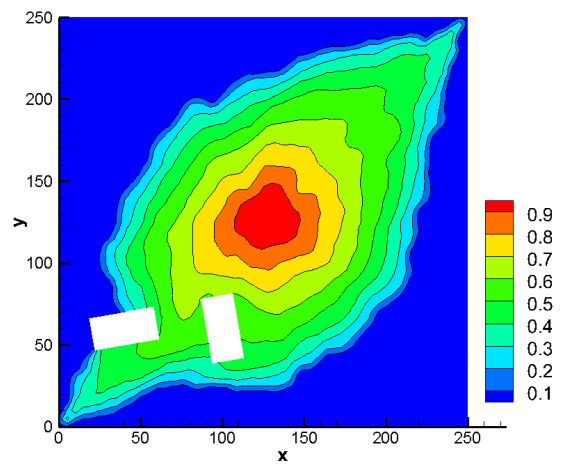

(d) Heterogeneous media, $\frac{\mu_{w}}{\mu_{n}}=\frac{1}{4}, B_{c}=10$

FIG. 6. Wetting saturation at $t=500$ days in Example 2.

of the total compute times. Among the tested methods, the multiplicative field-split INB-NE results in the smallest number of global Newton iterations and generally the least total compute time. For the multiplicative field-split approach, the percentage of the eliminated components in each stage is fixed for all NE applications. The percentage for the coupled element-block approach is greater than the saturation component-wise approach because its bad subset includes more degrees of freedom of both variables in the associated elements and neighbors, so that they can be eliminated together to obtain a better updated solution.

Figure 7 shows the nonlinear residual history for global Newton iteration at different time steps obtained using INB and INB-NE for Case $2\left(B_{c}=0\right)$ and Case 3 $\left(B_{c}=8\right)$. It is seen that for Case 2, INB stagnates with a long period to reach the convergence criteria, while the INB-NE methods converge quickly with fewer than 10 Newton iterations. When the capillary effect is taken into account, the nonlinearity of the system increases. The classical INB fails in line search since the first time step. Comparatively, the INB-NE methods are more robust and the convergence rate does not decrease much from the case without capillary effect. Figure 8 shows the step length $\lambda_{k}$ at the $1^{\text {st }}$ time step obtained using INB and INB-NE for Case 3 and Case 5 . We note that INB and the saturation component-wise INB-NE diverge for both 
TABLE 3

The average numbers of iterations and compute times obtained using INB and INB-NE for the heterogeneous media cases in Example 2 with different viscosity ratios and different capillary strength. '-' indicates that the case diverges for all time steps. ' $f$ ' means that the case diverges for the first time step. 'Pct.' denotes the mean percentage of the eliminated components per NE application.

\begin{tabular}{|c|c|c|c|c|c|c|c|c|c|c|c|c|}
\hline Case & $\mu_{w} / \mu_{n}$ & $B_{c}$ & $\varrho$ & Lag & $\mathrm{NI}_{g}$ & $\mathrm{LI}_{g}$ & $\mathrm{~T}_{t}$ & $\mathrm{~N}_{\mathrm{NE}}$ & $\mathrm{NI}_{\mathrm{NE}}$ & $\mathrm{LI}_{\mathrm{NE}}$ & $\mathrm{T}_{\mathrm{NE}}$ & Pct. \\
\hline & & & INB & & & & & & & & & \\
\hline 1 & $1 / 1$ & 0 & & & 22.8 & 7.9 & 4.44 & & & & & \\
\hline 2 & $1 / 2$ & 0 & & & 23.6 & 8.8 & 4.47 & & & & & \\
\hline 3 & $1 / 2$ & 8 & & & - & - & - & & & & & \\
\hline 4 & $1 / 4$ & 10 & & & 22.9 & 9.6 & 4.58 & & & & & \\
\hline \multirow[t]{2}{*}{5} & $1 / 4$ & 18 & & & - & - & - & & & & & \\
\hline & & & \multicolumn{10}{|c|}{ INB-NE (Saturation component-wise [50]) } \\
\hline $1 \dagger$ & $1 / 1$ & 0 & 0.005 & $\infty$ & 9.3 & 12.7 & 3.44 & 2.9 & 5.7 & 6.7 & 1.60 & $0.17 \%$ \\
\hline $2 \dagger$ & $1 / 2$ & 0 & 0.005 & $\infty$ & 10.5 & 12.0 & 3.30 & 2.7 & 5.1 & 6.5 & 1.31 & $0.18 \%$ \\
\hline $3 \dagger$ & $1 / 2$ & 8 & 0 & $\infty$ & 11.7 & 11.2 & 4.46 & 2.8 & 5.3 & 5.9 & 1.45 & $1.7 \%$ \\
\hline 4 & $1 / 4$ & 10 & 0 & 2 & 8.2 & 14.0 & 3.73 & 3.0 & 4.8 & 8.4 & 2.06 & $1.8 \%$ \\
\hline 5 & $1 / 4$ & 18 & 0 & 1 & - & - & - & - & - & - & - & - \\
\hline & & & \multicolumn{10}{|c|}{ INB-NE (Multiplicative field-split) } \\
\hline 1 & $1 / 1$ & 0 & & $\infty$ & 6.2 & 9.2 & 3.07 & 3.5 & 5.6 & 5.6 & 1.81 & $77.1 \% \backslash 22.9 \%$ \\
\hline 2 & $1 / 2$ & 0 & & $\infty$ & 7.9 & 12.1 & 3.35 & 3.5 & 5.7 & 5.7 & 1.80 & $77.1 \% \backslash 22.9 \%$ \\
\hline 3 & $1 / 2$ & 8 & & $\infty$ & 5.9 & 11.7 & 3.11 & 3.6 & 5.8 & 5.4 & 1.90 & $77.1 \% \backslash 22.9 \%$ \\
\hline 4 & $1 / 4$ & 10 & & 4 & 7.9 & 15.6 & 3.41 & 3.2 & 5.0 & 5.7 & 1.78 & $77.1 \% \backslash 22.9 \%$ \\
\hline 5 & $1 / 4$ & 18 & & 2 & 7.2 & 12.1 & 5.20 & 3.8 & 6.9 & 7.0 & 3.69 & $77.1 \% \backslash 22.9 \%$ \\
\hline & & & \multicolumn{10}{|c|}{ INB-NE (Coupled element-block) } \\
\hline 1 & $1 / 1$ & 0 & 0.05 & $\infty$ & 8.6 & 13.3 & 3.40 & 3.0 & 4.6 & 2.4 & 1.34 & $9.7 \%$ \\
\hline 2 & $1 / 2$ & 0 & 0.05 & $\infty$ & 8.9 & 13.4 & 3.24 & 3.0 & 4.6 & 2.3 & 1.39 & $9.6 \%$ \\
\hline 3 & $1 / 2$ & 8 & 0.01 & $\infty$ & 10.4 & 12.2 & 3.58 & 3.1 & 5.4 & 2.8 & 1.49 & $30.8 \%$ \\
\hline 4 & $1 / 4$ & 10 & 0.01 & 4 & 10.1 & 15.2 & 3.68 & 3.0 & 5.1 & 3.2 & 1.63 & $32.1 \%$ \\
\hline 5 & $1 / 4$ & 18 & 0.01 & 2 & 14.7 & 13.1 & 5.83 & 3.1 & 6.5 & 3.1 & 2.74 & $35.4 \%$ \\
\hline
\end{tabular}

cases with $\lambda_{k}$ less than 0.1, while the proposed INB-NE methods result in mostly full step $\lambda_{k}=1$, which implies fast convergence of the Newton iteration. To study the performance of inner Newton iteration for different approaches, we show the nonlinear residual history for the first application of NE for Case 5 in Figure 9. The saturation component-wise approach fails in line search at the $3^{\text {rd }}$ inner Newton step. On the other hand, the proposed approaches succeed to reduce the residual by a factor of 10 , which is sufficient for the subspace correction.

To see how the proposed NE preconditioners improve the convergence of the global Newton iteration, we show in Figure 10 the residual surface plots of the saturation components (left) and the pressure components (right) obtained using different INBNE methods for Case 5. Note that the data range is reset in Figure 10(e)-(h) for better visualization. We plot the residuals before and after the first application of NE where the saturation component-wise approach fails to converge for the inner Newton. At this moment, the high nonlinearities are confined to the vicinity of the injector before NE. For the saturation component-wise approach, the saturation residuals remain the same order of magnitude after NE, while the pressure residuals are barely changed. For the proposed NE approaches, these high nonlinearities are effectively reduced by a factor of nearly 100 as shown in Figure 10(e)-(h). Hence, the overall nonlinearities of the system are balanced properly and that leads to the convergence of the global 


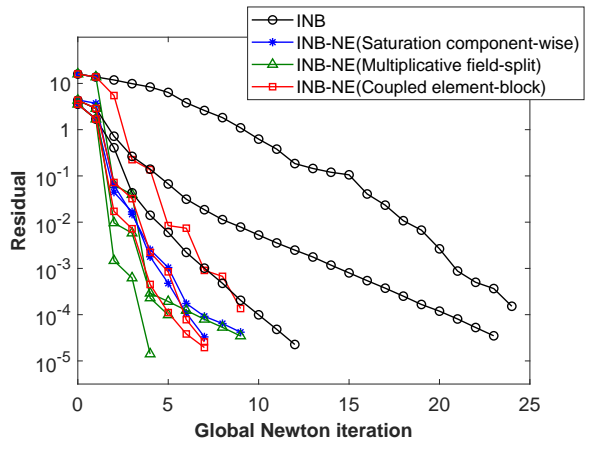

(a) Case $2\left(\mu_{w} / \mu_{n}=1 / 2, B_{c}=0\right)$

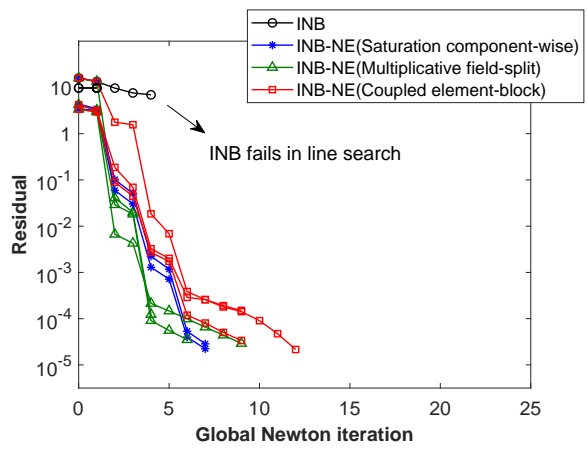

(b) Case $3\left(\mu_{w} / \mu_{n}=1 / 2, B_{c}=8\right)$

FIG. 7. Nonlinear residual history for global Newton iteration at the $1^{\text {st }}, 5^{\text {th }}, 10^{\text {th }}$ time steps obtained using INB and INB-NE in Example 2.

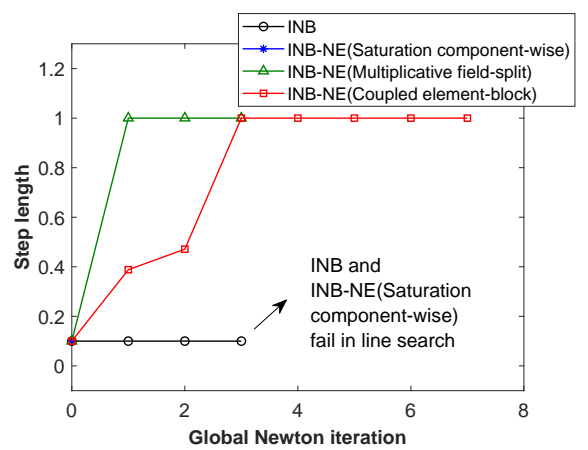

(a) Case $3\left(\mu_{w} / \mu_{n}=1 / 2, B_{c}=8\right)$

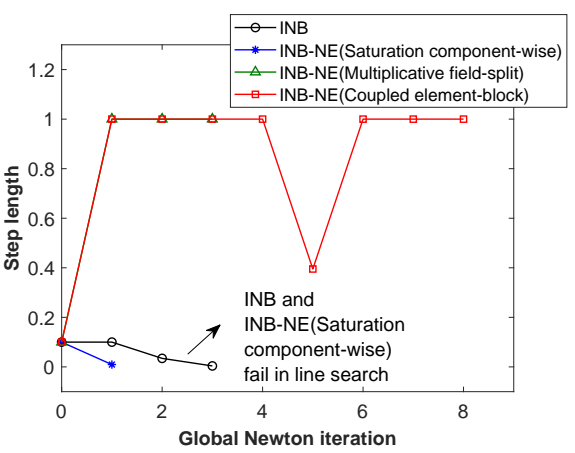

(b) Case $5\left(\mu_{w} / \mu_{n}=1 / 4, B_{c}=18\right)$

FIG. 8. Step length $\lambda_{k}$ at the $1^{\text {st }}$ time step obtained using INB and INB-NE in Example 2.

Newton iteration.

Next, we study the distribution of the bad components during the time stepping. Figure 11 shows the dynamics of the saturation and the corresponding residual surface plots at two different time steps obtained using the coupled element-block INB-NE method for Case 2. It is observed from Figure 11 (c)-(d) that the major bad components appear at the advancing fronts and they change with the propagation of the displacing fluid. As expected, the local high nonlinearities are identified and removed adaptively by the proposed NE preconditioner.

4.3. Example 3: A modified SPE Tenth case. In this example, a typical reservoir simulation process, the SPE Tenth case [12], is modified and simulated by using the proposed INB-NE methods. The data sets of porosity and absolute permeability $\mathbf{K}=\operatorname{diag}\left(K_{x x}, K_{y y}, K_{z z}\right)$ of the problem are highly heterogeneous, as shown in Figure 12. An injector is located in the middle of the domain and four producers are located on the four corners, respectively. All boundaries are impermeable $\left(\Gamma_{0}\right)$. A uniform mesh consisting of $16 \times 56 \times 22$ elements is used for the test, leading to 689,920 degrees of freedom. We load the rock data to the mesh points and adjust the zero porosity to the minimum positive one. Other relevant parameters are provided in Table 4. The coupled element-block INB-NE method is used for the simulation, and the preselected parameters are given as $\rho_{0}=0.5, \varepsilon=10^{-4}, \varrho=0.05$, and $\delta_{n}=1$. An 


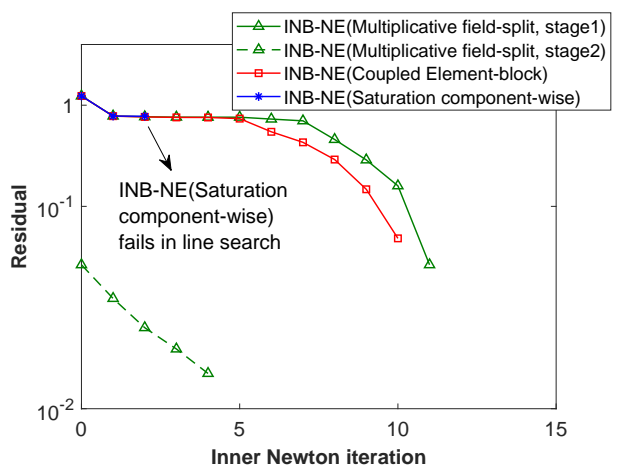

FIG. 9. Nonlinear residual history for inner Newton iteration in the first application of NE obtained using different approaches for Case 5 in Example 2.

adaptive time step size is used where $\Delta t$ is rescaled by 0.8 if the global Newton does not converge within 15 iterations, and is rescaled by 1.1 if the global Newton converges within 7 iterations. $\Delta t$ is also limited by a maximum time step size $\Delta t_{\max }=2$ day. The initial time step size is 1 day. The simulation is carried out using 1,024 processor cores. Figure 13 shows the wetting saturation at $t=850$ days for the top and bottom layers of the porous media. The two layers display different main flow directions due to the heterogeneity of the rock data.

TABLE 4

Relevant parameters for Example 3

\begin{tabular}{ll}
\hline Domain dimensions & $365.76 \mathrm{~m} \times 670.56 \mathrm{~m} \times 51.816 \mathrm{~m}$ \\
Rock properties & $\phi \in\left[10^{-6}, 0.5\right], K_{z z} \in\left[7.7 \times 10^{-8}, 6 \times 10^{3}\right] \mathrm{mD}$ \\
& $K_{x x}=K_{y y} \in\left[7.7 \times 10^{-4}, 2 \times 10^{4}\right] \mathrm{mD}$ \\
Fluid properties & $\mu_{w}=0.3 \mathrm{cP}, \mu_{n}=3 \mathrm{cP}$ \\
& $\rho_{w}=1025 \mathrm{~kg} / \mathrm{m}^{3}, \rho_{n}=849 \mathrm{~kg} / \mathrm{m}^{3}$ \\
Relative permeabilities & $\beta=2$ in $(2.8)$ \\
Capillary pressure & $\bar{B}_{c}=0 \mathrm{in}(2.8)$ \\
Residual saturations & $s_{r w}=0.2, s_{r n}=0.2$ \\
Injection rate & $61.6 \mathrm{~m}^{3} /$ day \\
Production well & $r_{w}=0.125 \mathrm{~m}, s_{k}=0, p_{b h}=4000 \mathrm{psi}[10]$ \\
\hline
\end{tabular}

To understand the impact of the parameters on the performance of the NE preconditioner, we test the coupled element-block approach with different values of $\varrho$, $\delta_{n}, \gamma_{r}^{\mathrm{NE}}$, as well as different recomputed frequency for the subspace Jacobian and preconditioner. The time step size is fixed to $\Delta t=1$ day. In Table 5 , we show the effect of these parameters on the number of nonlinear and linear iterations, as well as the compute time. When $\varrho$ decreases or $\delta_{n}$ increases, the number of bad components increases, in general the cost for solving subspace nonlinear problems also increases. Paying such a price helps reduce the number of global Newton iterations. We find from Table 5 that the pair of values $\left(\varrho, \delta_{n}\right)=(0.05,1)$ is preferable to minimize the total compute time. On the other hand, the relative tolerance $\gamma_{r}^{\mathrm{NE}}$ is used to determine how accurately the subspace nonlinear problem is solved. The results indicate that using a smaller $\gamma_{r}^{\mathrm{NE}}$ slightly reduces the the number of global Newton iterations, but the total compute time increases considerably. For the inner Newton iteration, we can reuse the subspace Jacobian and preconditioner for several iterations in order to reduce the inner compute time. This is confirmed when we increase the lagging 


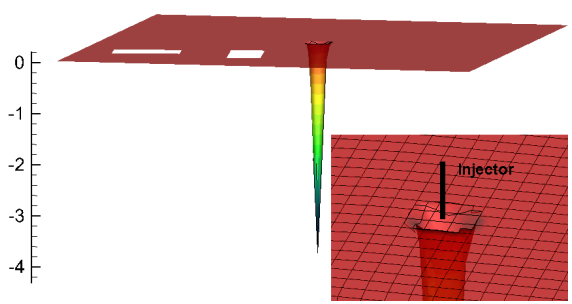

(a) Before NE

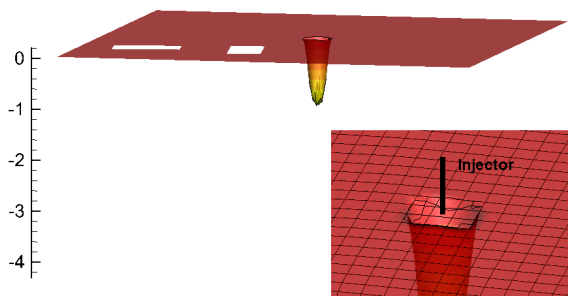

(c) After the saturation component-wise NE

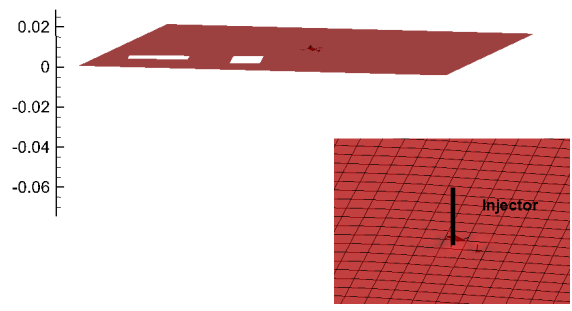

(e) After the multiplicative field-split NE

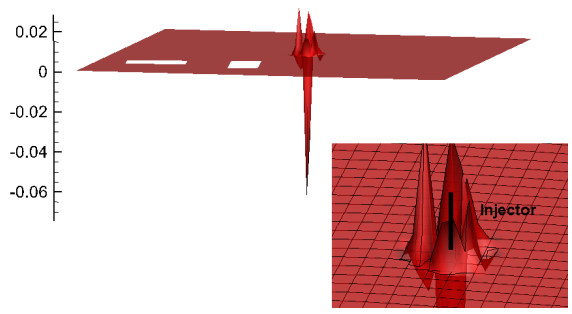

(g) After the coupled element-block NE

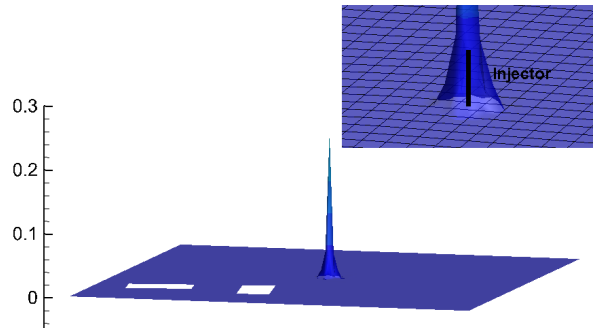

(b) Before NE

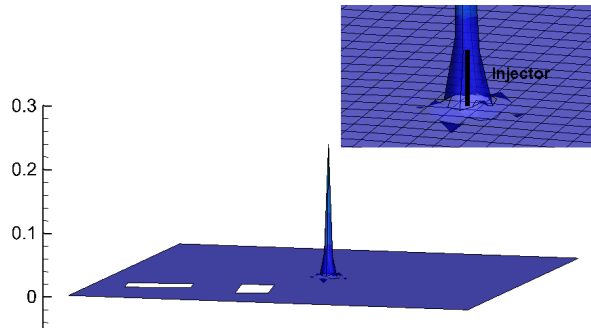

(d) After the saturation component-wise NE
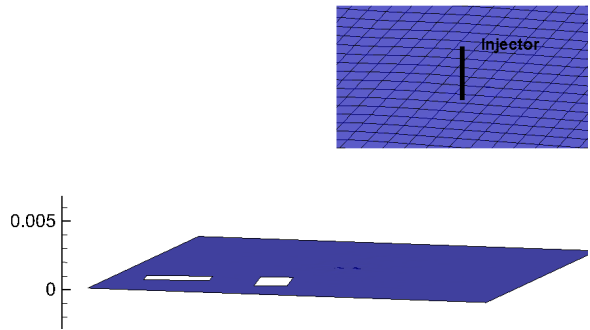

(f) After the multiplicative field-split NE


(h) After the coupled element-block NE

FIG. 10. Results at the $2^{\text {nd }}$ global Newton iteration at the $1^{\text {st }}$ time step obtained using INB-NE for Case 5 in Example 2. (left) Residual of saturation components; (right) Residual of pressure components. Note that the data range is reset in (e)-(h) for better visualization.

number from 1 to $\infty$, and the total compute time can be saved too. Note that the lagging in inner Newton barely changes the number of global Newton iterations.

We further compare the performance of INB and INB-NE with different flow rates. The above optimal parameters are used for the coupled element-block approach. For comparison, we use the same values for the common parameters in the other two $\mathrm{NE}$ approaches, and a different value of $\varrho=0.01$ for the saturation component-wise 


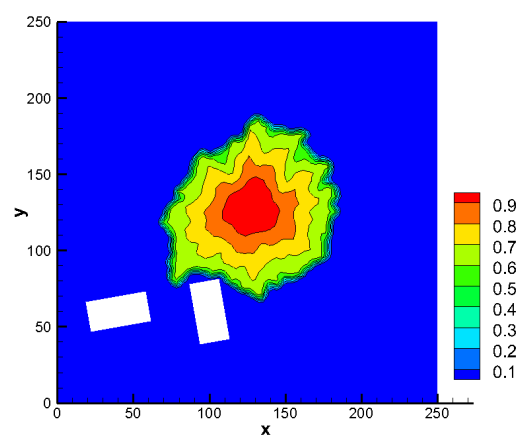

(a) Wetting saturation

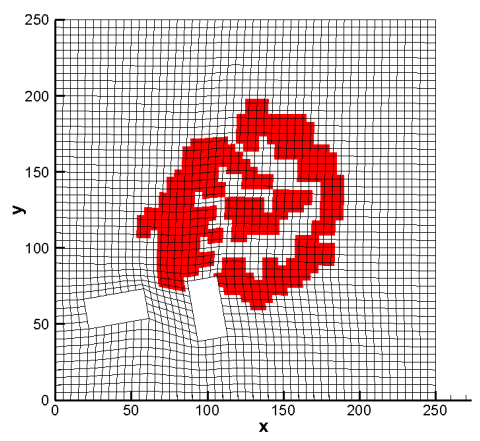

(c) Bad subset

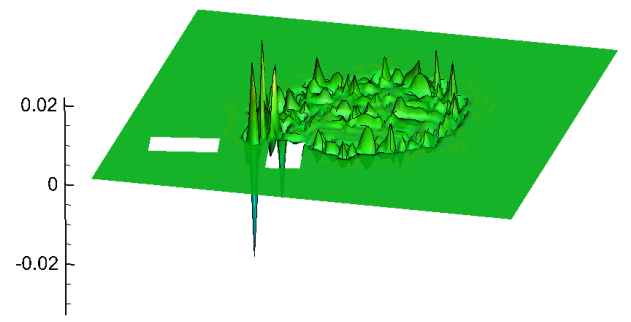

(e) Residual before NE

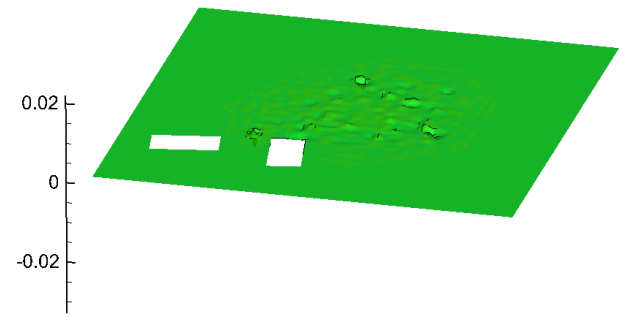

(g) Residual after NE

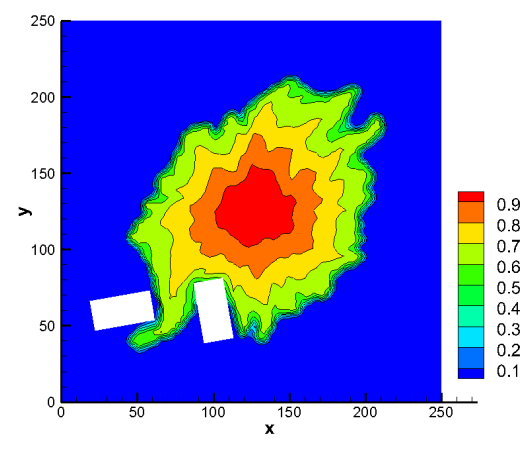

(b) Wetting saturation

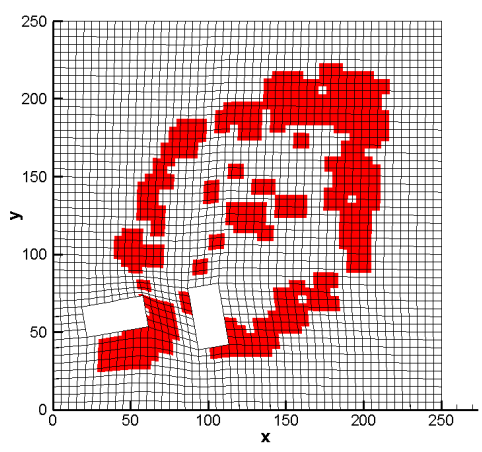

(d) Bad subset

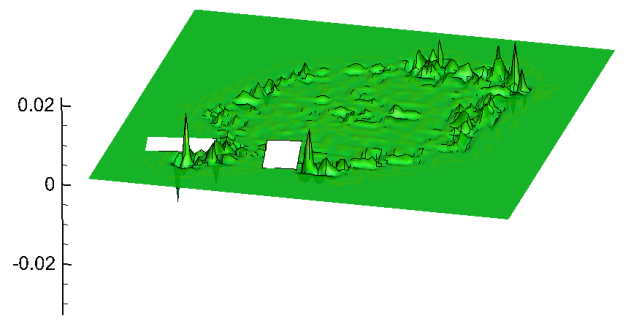

(f) Residual before NE

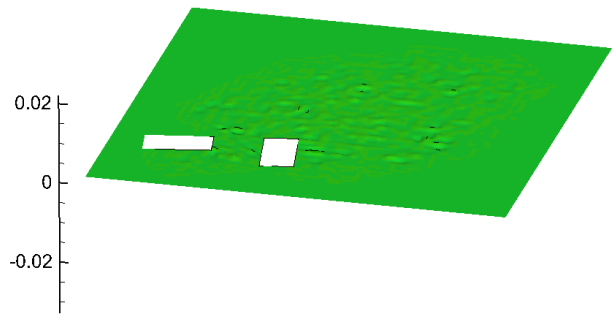

(h) Residual after NE

FIG. 11. Results at the $2^{\text {nd }}$ global Newton iteration at the $200^{\text {th }}$ time step (left) and the $400^{\text {th }}$ time step (right) obtained using the coupled element-block INB-NE method for Case 2 in Example 2. In (c) and (d), the bad subset $\mathcal{E}_{k}^{b}$ is composed of the elements fully inside the red region. (e)-( $h$ ) Residual of saturation components. 


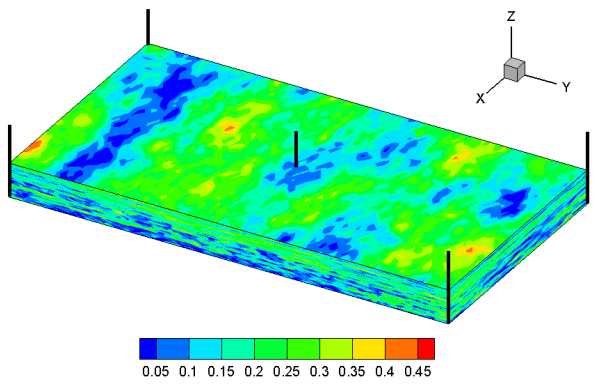

(a) Porosity

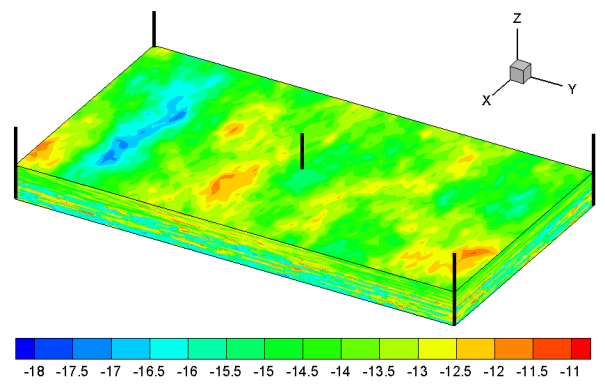

(b) Permeability $\left(\log _{10} K_{y y}\right)$

Fig. 12. Example 3: Porosity and the y-direction permeability in a modified SPE Tenth case.

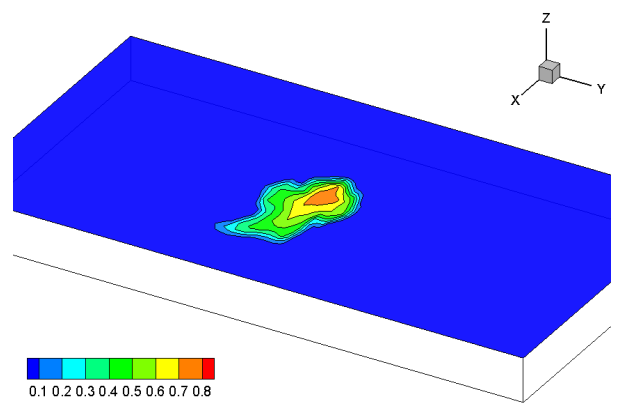

(a) Top layer

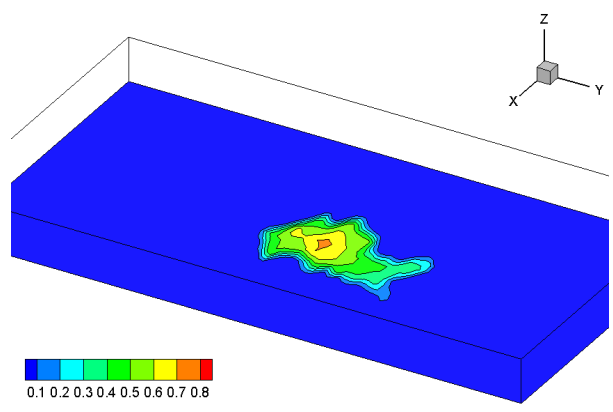

(b) Bottom layer

FIG. 13. Slice view of wetting saturation at $t=850$ days in Example 3.

TABLE 5

Impact of pre-selected parameters on the coupled element-block INB-NE method in Example 3. The flow rate is $61.6 \mathrm{~m}^{3} /$ day. The time step size is $\Delta t=1$ day.

\begin{tabular}{lcccccccccc}
\hline$\varrho$ & $\delta_{n}$ & $\gamma_{r}^{\mathrm{NE}}$ & $\mathrm{Lag}$ & $\mathrm{NI}_{g}$ & $\mathrm{LI}_{g}$ & $\mathrm{~T}_{t}$ & $\mathrm{~N}_{\mathrm{NE}}$ & $\mathrm{NI}_{\mathrm{NE}}$ & $\mathrm{LI}_{\mathrm{NE}}$ & $\mathrm{T}_{\mathrm{NE}}$ \\
\hline 0.2 & 1 & $10^{-1}$ & $\infty$ & 8.5 & 294.7 & 22.29 & 2.4 & 5.3 & 2.5 & 3.70 \\
0.1 & 1 & $10^{-1}$ & $\infty$ & 7.7 & 174.5 & 18.98 & 2.3 & 4.5 & 3.8 & 3.80 \\
0.05 & 1 & $10^{-1}$ & $\infty$ & 7.0 & 182.5 & 15.79 & 2.4 & 4.0 & 5.3 & 3.59 \\
0.025 & 1 & $10^{-1}$ & $\infty$ & 5.8 & 213.5 & 22.65 & 2.5 & 3.9 & 7.1 & 4.09 \\
\hline \multicolumn{8}{c}{ Impact of $\delta_{n}$} \\
\hline 0.05 & 0 & $10^{-1}$ & $\infty$ & 7.2 & 214.6 & 24.24 & 2.5 & 4.6 & 3.8 & 5.32 \\
0.05 & 2 & $10^{-1}$ & $\infty$ & 6.1 & 193.5 & 22.90 & 2.4 & 4.0 & 5.9 & 3.99 \\
0.05 & 3 & $10^{-1}$ & $\infty$ & 5.8 & 198.1 & 26.64 & 2.6 & 4.0 & 5.4 & 4.84 \\
\hline \multicolumn{8}{c}{ Impact of $\gamma_{r}^{\mathrm{NE}}$} \\
\hline 0.05 & 1 & $10^{-2}$ & $\infty$ & 5.7 & 223.1 & 16.77 & 1.6 & 6.4 & 8.0 & 3.93 \\
0.05 & 1 & $10^{-3}$ & $\infty$ & 5.0 & 346.8 & 20.43 & 1.4 & 9.9 & 7.2 & 4.11 \\
0.05 & 1 & $10^{-4}$ & $\infty$ & 4.7 & 333.6 & 25.89 & 1.1 & 10.9 & 9.7 & 5.81 \\
\hline \multicolumn{1}{c}{ Impact of lagging subspace Jacobian and preconditioner } \\
\hline 0.05 & 1 & $10^{-1}$ & 1 & 6.5 & 201.9 & 19.47 & 2.4 & 4.2 & 5.2 & 7.43 \\
0.05 & 1 & $10^{-1}$ & 2 & 6.8 & 207.2 & 17.03 & 2.3 & 3.8 & 6.0 & 4.48 \\
0.05 & 1 & $10^{-1}$ & 4 & 6.7 & 212.0 & 16.52 & 2.4 & 3.1 & 6.4 & 3.62 \\
\hline
\end{tabular}


approach to obtain optimal performance. Table 6 summarizes the results obtained using a fixed time step size $\Delta t=1$ day. Figure 14 shows the nonlinear residual history for different time steps. From the table and the figure, we see that the number of global Newton iterations and the total compute time obtained using INB increase notably with the increase of the flow rate. Using the NE preconditioner significantly improves the robustness and efficiency of INB. Specifically, the multiplicative fieldsplit approach results in the smallest number of global Newton iterations, while the coupled element-block approach results in the least total compute time. One distinct feature among the approaches is that the coupled element-block approach requires fewer linear iterations in the NE step than the other two field-split approaches, such a result is also observed in Example 2. The physically coupled approach groups all variables associated with an element into a subsystem and solves them simultaneously. As demonstrated in $[27,46]$, this usually yields better linear convergence when the subsystems involve locally stiff coefficients or the physical variables change abruptly in the local region.

TABLE 6

The average numbers of iterations and compute times obtained using INB and INB-NE with different flow rates in Example 3. The time step size is $\Delta t=1$ day.

\begin{tabular}{|c|c|c|c|c|c|c|c|}
\hline Flow rate & $\mathrm{NI}_{g}$ & $\mathrm{LI}_{g}$ & $\mathrm{~T}_{t}$ & $\mathrm{~N}_{\mathrm{NE}}$ & $\mathrm{NI}_{\mathrm{NE}}$ & $\mathrm{LI}_{\mathrm{NE}}$ & $\mathrm{T}_{\mathrm{NE}}$ \\
\hline$\left(\mathrm{m}^{3} /\right.$ day $)$ & INB & & & & & & \\
\hline 41.1 & 9.5 & 248.7 & 17.82 & & & & \\
\hline 61.6 & 23.2 & 185.4 & 31.29 & & & & \\
\hline & INB & $\mathrm{NE}(\mathrm{S}$ & turatic & n cor & ponen & -wise & 50]) \\
\hline 41.1 & 5.2 & 384.7 & 17.83 & 1.4 & 2.9 & 67.5 & 3.62 \\
\hline 61.6 & 5.9 & 217.5 & 20.43 & 2.3 & 5.4 & 60.1 & 8.60 \\
\hline & \multicolumn{7}{|c|}{ INB-NE (Multiplicative field-split) } \\
\hline 41.1 & $\begin{array}{ll}3.1 \\
\end{array}$ & 377.0 & 15.34 & 2.6 & 3.3 & 56.8 & 6.66 \\
\hline 61.6 & 3.5 & 230.3 & 18.62 & 3.3 & 6.2 & 48.0 & 11.95 \\
\hline & \multicolumn{7}{|c|}{ INB-NE (Coupled element-block) } \\
\hline 41.1 & 5.0 & 272.4 & 13.62 & 1.5 & 2.7 & 6.6 & 1.97 \\
\hline 61.6 & 7.0 & 182.5 & 15.79 & 2.4 & 4.0 & 5.3 & 3.59 \\
\hline
\end{tabular}

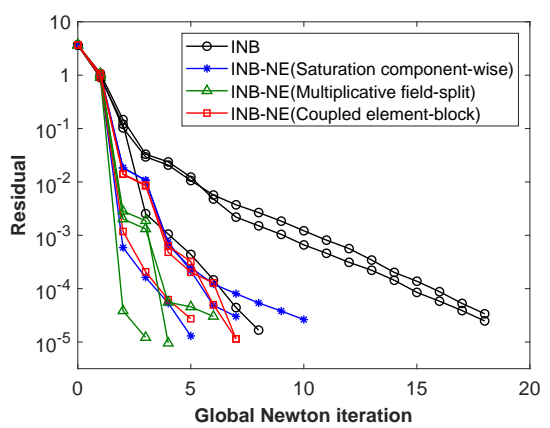

(a) Flow rate: $41.1 \mathrm{~m}^{3} /$ day

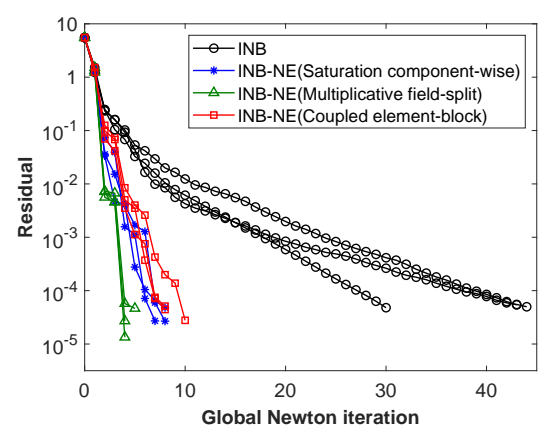

(b) Flow rate: $61.6 \mathrm{~m}^{3} /$ day

FIG. 14. Nonlinear residual history for global Newton iteration at the $6^{\text {th }}, 8^{\text {th }}, 10^{\text {th }}$ time steps obtained using INB and INB-NE in Example 3. The time step size is $\Delta t=1$ day.

In the following, we study the parallel performance of INB and INB-NE for Example 3. In the tests, the problem size is fixed to 689,920 degrees of freedom and we 
vary the number of processor cores from 256 to 2,048. To obtain the best performance, we use the above optimal parameters for various approaches. For the linear solver in both the global and subspace Jacobian systems, the size of overlap is $\delta_{l}=1$ and the subdomain solver is $\operatorname{ILU}(2)$. The flow rate is fixed to $61.6 \mathrm{~m}^{3}$ /day and the time step size is $\Delta t=1$ day. Table 7 lists the scalability results in detail. Figure 15 shows the change of total compute time with respect to the number of processor cores. As $n p$ increases, the numbers of global and inner Newton iterations remain stable, while the total compute times and the compute times for the NE preconditioner decrease correspondingly. A superlinear speedup is observed when $n p=512$ for the classical INB and the proposed INB-NE methods. The final speedup of the proposed INB-NE methods reaches roughly $5.6 \times$ when $n p$ is 8 -fold, which shows reasonably good scalability. We note that the total compute times for the proposed INB-NE are essentially half of the classical INB.

TABLE 7

Scalability results of INB and INB-NE for Example 3 with flow rate $61.6 \mathrm{~m}^{3} /$ day. The system has 689,920 degrees of freedom. The size of overlap is $\delta_{l}=1$ and the subdomain solver is ILU(2). The time step size is $\Delta t=1$ day.

\begin{tabular}{|c|c|c|c|c|c|c|c|}
\hline \multirow[t]{2}{*}{$n p$} & $\mathrm{NI}_{g}$ & $\mathrm{LI}_{g}$ & $\mathrm{~T}_{t}$ & $\mathrm{~N}_{\mathrm{NE}}$ & $\mathrm{NI}_{\mathrm{NE}}$ & $\mathrm{LI}_{\mathrm{NE}}$ & $\mathrm{T}_{\mathrm{NE}}$ \\
\hline & \multicolumn{7}{|l|}{ INB } \\
\hline 256 & 23.4 & 91.6 & 154.64 & & & & \\
\hline 512 & 23.7 & 136.9 & 51.26 & & & & \\
\hline 1,024 & 23.2 & 185.4 & 31.29 & & & & \\
\hline \multirow[t]{2}{*}{2,048} & 24.6 & 194.7 & 21.82 & & & & \\
\hline & \multicolumn{7}{|c|}{ INB-NE (Saturation component-wise [50]) } \\
\hline 256 & 6.2 & 145.9 & 68.02 & 1.9 & 4.3 & 45.3 & 20.05 \\
\hline 512 & 6.1 & 251.0 & 35.37 & 2.4 & 5.4 & 48.5 & 13.14 \\
\hline 1,024 & 5.9 & 217.5 & 20.43 & 2.3 & 5.4 & 60.1 & 8.60 \\
\hline \multirow[t]{2}{*}{2,048} & 6.8 & 206.7 & 13.72 & 2.4 & 5.4 & 57.2 & 5.67 \\
\hline & \multicolumn{7}{|c|}{ INB-NE (Multiplicative field-split) } \\
\hline 256 & 4.0 & 114.1 & 65.04 & 3.2 & 6.4 & 30.8 & 35.99 \\
\hline 512 & 3.7 & 150.5 & 28.64 & 3.2 & 7.2 & 34.7 & 18.42 \\
\hline 1,024 & 3.5 & 230.3 & 18.62 & 3.3 & 6.2 & 48.0 & 11.95 \\
\hline \multirow[t]{2}{*}{2,048} & 3.9 & 311.2 & 12.10 & 2.9 & 5.8 & 40.6 & 6.25 \\
\hline & \multicolumn{7}{|c|}{ INB-NE (Coupled element-block) } \\
\hline 256 & 6.8 & 130.7 & 65.23 & 2.2 & 4.0 & 4.6 & 15.11 \\
\hline 512 & 6.1 & 177.7 & 23.38 & 2.3 & 3.7 & 4.6 & 5.26 \\
\hline 1,024 & 7.0 & 182.5 & 15.79 & 2.4 & 4.0 & 5.3 & 3.59 \\
\hline 2,048 & 6.6 & 295.2 & 11.65 & 2.0 & 4.3 & 5.2 & 2.07 \\
\hline
\end{tabular}

5. Concluding remarks. We develop a class of nonlinearly preconditioned inexact Newton methods for the simulation of two-phase flows in porous media of high contrast. The model of two-phase flow is discretized by a fully implicit discontinuous Galerkin finite element method. When a classical inexact Newton method with backtracking is used to solve the resulting nonlinear system, it often suffers from slow convergence or failure in line search. In this work, we propose two nonlinear elimination preconditioning strategies to handle this issue. The key idea is to perform subspace correction to remove the local high nonlinearities that cause difficulty for the convergence. One strategy is based on the field-splitting of pressure and saturation, the other strategy is based on the field-coupling of the two variables. We test the algorithms using several nonlinearly difficult flow problems in heterogeneous media. Numerical experiments show that the proposed methods are more robust and faster 




FIG. 15. Parallel performance of INB and INB-NE for Example 3 with flow rate $61.6 \mathrm{~m}^{3} /$ day. The system has 689,920 degrees of freedom. The size of overlap is $\delta_{l}=1$ and the subdomain solver is $I L U(2)$. The time step size is $\Delta t=1$ day.

than the existing methods with respect to some physical and numerical parameters, and is scalable on a supercomputer with thousands of processor cores. Although the discussion of the paper is restricted to the two-phase flow problems, the algorithms are expected to work well for other nonlinear models in reservoir simulation.

[1] T. Arbogast, M. Juntunen, J. Pool, and M. F. Wheeler, A discontinuous Galerkin method for two-phase flow in a porous medium enforcing $H($ div) velocity and continuous capillary pressure, Comput. Geotech., 17 (2013), pp. 1055-1078.

[2] S. Balay, S. Abhyankar, M. F. Adams, J. Brown, P. Brune, K. Buschelman, L. Dalcin, V. Eijkhout, W. D. Gropp, D. Kaushik, M. G. Knepley, D. A. May, L. C. Mcinnes, R. T. Mills, T. Munson, K. Rupp, P. Sanan, B. F. Smith, S. Zampini, H. Zhang, and H. Zhang, PETSc Users Manual, Argonne National Laboratory, 2020.

[3] P. Bastian, A fully-coupled discontinuous Galerkin method for two-phase flow in porous media with discontinuous capillary pressure, Comput. Geosci., 18 (2014), pp. 779-796.

[4] P. Brune, M. Knepley, B. Smith, And X. Tu, Composing scalable nonlinear algebraic solvers, SIAM Review, 57 (2015), pp. 535-565.

[5] X.-C. Cai, W. D. Gropp, D. E. Keyes, R. G. Melvin, and D. P. Young, Parallel NewtonKrylov-Schwarz algorithms for the transonic full potential equation, SIAM J. Sci. Comput., 19 (1998), pp. 246-265.

[6] X.-C. CAi AND M. SARKIS, A restricted additive Schwarz preconditioner for general sparse linear systems, SIAM J. Sci. Comput., 21 (1999), pp. 792-797.

[7] X.-C. Cai and D. E. Keyes, Nonlinearly preconditioned inexact Newton algorithms, SIAM J. Sci. Comput., 24 (2002), pp. 183-200.

[8] X.-C. CAI AND X. LI, Inexact Newton methods with restricted additive Schwarz based nonlinear elimination for problems with high local nonlinearity, SIAM J. Sci. Comput., 33 (2011), pp. $746-762$.

[9] Z. Chen, G. Huan, And B. Li, An improved IMPES method for two-phase flow in porous media, Transport Porous Med., 54 (2004), pp. 361-376.

[10] Z. Chen, G. Huan, And Y. Ma, Computational Methods for Multiphase Flows in Porous Media, SIAM, Philadelphia, PA, USA, 2006.

[11] H. Chen, J. Kou, S. Sun, And T. Zhang, Fully mass-conservative IMPES schemes for incompressible two-phase flow in porous media, Comput. Methods Appl. Mech. Engrg., 350 (2019), pp. 641-663.

[12] M. Christie And M. Blunt, Tenth SPE comparative solution project: A comparison of upscaling techniques, in: SPE Reservoir Simulation Symposium, Society of Petroleum Engineers, 2001, pp. 308-317. 
[13] C. N. Dawson, H. Klíe, M. F. Wheeler, and C. S. Woodward, A parallel, implicit, cellcentered method for two-phase flow with a preconditioned Newton-Krylov solver, Computat. Geosci., 1 (1997), pp. 215-249.

[14] J. E. Dennis And R. B. Schnabel, Numerical Methods for Unconstrained Optimization and Nonlinear Equations, SIAM, Philadelphia, 1996.

[15] S. C. Eisenstat and H. F. Walker, Choosing the forcing terms in an inexact Newton method, SIAM J. Sci. Comput., 17 (1996), pp. 16-32.

[16] Y. EPSHTEYN AND B. RIVIÈRE, Fully implicit discontinuous finite element methods for twophase flow, Appl. Numer. Math., 57 (2007), pp. 383-401.

[17] A. ERn, I. Mozolevski, ANd L. SChUH, Discontinuous Galerkin approximation of two-phase flows in heterogeneous porous media with discontinuous capillary pressures, Comput. Methods Appl. Mech. Engrg., 199 (2010), pp. 1491-1501.

[18] S. Gong AND X.-C. CAI, A nonlinear elimination preconditioned inexact Newton method for heterogeneous hyperelasticity, SIAM J. Sci. Comput., 41 (2019), pp. S390-S408.

[19] S. Gries, K. Stüben, G. L. Brown, D. Chen, and D. A. Collins, Preconditioning for efficiently applying algebraic multigrid in fully implicit reservoir simulations, SPE J., 19 (2014), pp. 726-736.

[20] W. D. Gropp, D. K. Kaushik, D. E. Keyes, and B. F. Smith, High-performance parallel implicit CFD, Parallel Comput., 27 (2001), pp. 337-362.

[21] H. Hoteit AND A. FiroozABAdi, Numerical modeling of two-phase flow in heterogeneous permeable media with different capillarity pressures, Adv. Water Resour., 31 (2008), pp. 56-73.

[22] J. HuAng, C. YANG, AND X.-C. CAI, A nonlinearly preconditioned inexact Newton algorithm for steady lattice Boltzmann equations, SIAM J. Sci. Comput., 38 (2016), pp. 1701-1724.

[23] F.-N. Hwang AND X.-C. CAI, A parallel nonlinear additive Schwarz preconditioned inexac$t$ Newton algorithm for incompressible Navier-Stokes equations, J. Comput. Phys., 204 (2005), pp. 666-691.

[24] F.-N. Hwang And X.-C. CAI, A class of parallel two-level nonlinear Schwarz preconditioned inexact Newton algorithms, Comput. Meth. Appl. Mech. Eng., 196 (2007), pp. 1603-1611.

[25] F.-N. Hwang , H.-L. Lin, AND X.-C. CAI, Two-level nonlinear elimination-based preconditioners for inexact Newton methods with application in shoched duct flow calculation, Electron. Trans. Numer. Anal., 37 (2010), pp. 239-251.

[26] F.-N. Hwang, Y.-C. Su, AND X.-C. CAI, A parallel adaptive nonlinear elimination preconditioned inexact Newton method for transonic full potential equation, Comput. Fluids, 110 (2015), pp. 96-107.

[27] V. John AND L. TOBISKA, Numerical performance of smoothers in coupled multigrid methods for the parallel solution of the incompressible Navier-Stokes equations, Int. J. Numer. Meth. Fluids, 33 (2000), pp. 453-473.

[28] B. S. Kirk, J. W. Peterson, R. H. Stogner, and G. F. Carey, libMesh: A C++ library for parallel adaptive mesh refinement/coarsening simulations, Eng. Comput., 22 (2006), pp. 237-254.

[29] D. A. Knoll and D. E. Keyes, Jacobian-free Newton-Krylov methods: A survey of approaches and applications, J. Comput. Phys., 193 (2004), pp. 357-397.

[30] J. Kou AND S. Sun, Upwind discontinuous Galerkin methods with conservation of mass of both phases for incompressible two-phase flow in porous media, Numer. Methods Partial Differ. Equ., 30 (2014), pp. 1674-1699.

[31] R. LI, H. YANG, AND C. YANG, Parallel multilevel restricted Schwarz preconditioners for implicit simulation of subsurface flows with Peng-Robinson equation of state, J. Comput. Phys., 422 (2020), 109745.

[32] K. A. LiE, An Introduction to Reservoir Simulation Using MATLAB/GNU Octave: User Guide for the MATLAB Reservoir Simulation Toolbox (MRST), Cambridge: Cambridge University Press, 2019.

[33] L. Liu And D. E. Keyes, Field-split preconditioned inexact Newton algorithms, SIAM J. Sci. Comput., 37 (2015), pp. 1388-1409.

[34] H. Liu, K. WANG, AND Z. Chen, A family of constrained pressure residual preconditioners for parallel reservoir simulations, Numer. Linear Algebra Appl., 23 (2016), pp. 120-146.

[35] L. LiU AND D. E. Keyes, Convergence analysis for the multiplicative Schwarz preconditioned inexact Newton algorithm, SIAM J. Numer. Anal., 54 (2016), pp. 3145-3166.

[36] L. Liu, D. E. Keyes, And R. Krause, A note on adaptive nonlinear preconditioning techniques, SIAM J. Sci. Comput., 40 (2018), pp. 1171-1186.

[37] L. Luo, W.-S. Shiu, R. Chen, AND X.-C. CAI, A nonlinear elimination preconditioned inexact Newton method for blood flow problems in human artery with stenosis, J. Comput. Phys., 399 (2019), 108926. 
[38] L. Luo, L. Liu, X.-C. CAI, And D. E. Keyes, Fully implicit hybrid two-level domain decomposition algorithms for two-phase flows in porous media on $3 D$ unstructured grids, J. Comput. Phys., 409 (2020), 109312.

[39] L. Luo, X.-C. CAI, Z. YAn, L. Xu, AND D. E. Keyes, A multilayer nonlinear elimination preconditioned inexact Newton method for steady-state incompressible flow problems in three dimensions, SIAM J. Sci. Comput., 42 (2020), B1404-B1428.

[40] A. Michel, A finite volume scheme for the simulation of two-phase incompressible flow in porous media, SIAM J. Numer. Analy., 41 (2003), pp. 1301-1317.

[41] J. E. P. Monteagudo and A. Firoozabadi, Comparison of fully implicit and IMPES formulations for simulation of water injection in fractured and unfractured media, Internat. J. Numer. Methods Engrg., 69 (2007), pp. 698-728.

[42] J. T. Oden, I. BABušKa, And C. E. BAumann, A discontinuous hp finite element method for diffusion problems, J. Comput. Phys., 146 (1998), pp. 491-519.

[43] Y. SAAD, Iterative Methods for Sparse Linear Systems, SIAM: Philadelphia, PA, 2003.

[44] J. N. Shadid, R. S. Tuminaro, and H. F. Walker, An inexact Newton method for fully coupled solution of the Navier-Stokes equations with heat and mass transport, J. Comput. Phys., 137 (1997), pp. 155-185.

[45] S. Sun AND M.F. WheELeR, Symmetric and nonsymmetric discontinuous Galerkin methods for reactive transport in porous media, SIAM J. Numer. Anal., 43 (2005), pp. 195-219.

[46] S. VANKA, Block-implicit multigrid calculation of two-dimensional recirculating flows, Comp. Meth. Appl. Mech. Eng., 59 (1986), pp. 29-48.

[47] K. WAng, L. Zhang, AND Z. Chen, Development of discontinuous Galerkin methods and a parallel simulator for reservoir simulation, SPE J., 2015.

[48] H. YAng, F.-N. HWANG, AND X.-C. CAI, Nonlinear preconditioning techniques for full-space Lagrange-Newton solution of PDE-constrained optimization problems, SIAM J. Sci. Comput., 38 (2016), pp. 2756-2778.

[49] H. YAng, C. YANG, AND S. Sun, Active-set reduced-space methods with nonlinear elimination for two-phase flow problems in porous media, SIAM J. Sci. Comput., 38 (2016), pp. 593618.

[50] H. YANG, S. Sun, ANd C. YANG, Nonlinearly preconditioned semismooth Newton methods for variational inequality solution of two-phase flow in porous media, J. Comput. Phys., 332 (2017), pp. 1-20.

[51] H. YANG AND F.-N. HwANG, An adaptive nonlinear elimination preconditioned inexact Newton algorithm for highly local nonlinear multicomponent PDE systems, Appl. Numer. Math., 133 (2018), pp. 100-115.

[52] H. YANG, Y. Li, AND S. Sun, Nonlinearly preconditioned constraint-preserving algorithms for subsurface three-phase flow with capillarity, Comput. Methods Appl. Mech. Eng., 367 (2020), 113140. 